\title{
SDDV: scalable data dissemination in vehicular ad hoc networks
}

\author{
Wim Vandenberghe ${ }^{1 *}$, Hans Cappelle ${ }^{2}$, Ingrid Moerman ${ }^{1}$ and Piet Demeester ${ }^{1}$
}

\begin{abstract}
An important challenge in the domain of vehicular ad hoc networks (VANET) is the scalability of data dissemination. Under dense traffic conditions, the large number of communicating vehicles can easily result in a congested wireless channel. In that situation, delays and packet losses increase to a level where the VANET cannot be applied for road safety applications anymore. This paper introduces scalable data dissemination in vehicular ad hoc networks (SDDV), a holistic solution to this problem. It is composed of several techniques spread across the different layers of the protocol stack. Simulation results are presented that illustrate the severity of the scalability problem when applying common state-of-the-art techniques and parameters. Starting from such a baseline solution, optimization techniques are gradually added to SDDV until the scalability problem is entirely solved. Besides the performance evaluation based on simulations, the paper ends with an evaluation of the final SDDV configuration on real hardware. Experiments including 110 nodes are performed on the iMinds w-iLab.t wireless lab. The results of these experiments confirm the results obtained in the corresponding simulations.
\end{abstract}

Keywords: Vehicular ad hoc network; VANET; Scalability problem; V2V; ITS

\section{Introduction}

Vehicular ad hoc networks (VANET) provide local vehicle-to-vehicle $(\mathrm{V} 2 \mathrm{~V})$ and vehicle-to-infrastructure (V2I) communications to support intelligent transportations systems (ITS) applications. Example applications are emergency electronic brake lights, slow vehicle indication, wrong way driver warning, stationary vehicle warning, lane change assistance, etc. The technology used to enable such ad hoc networking is called IEEE 802.11p [1]. This is an amendment to the IEEE 802.11 standard that contains several enhancements to improve performance under vehicular conditions. Examples are an increased output power, dedicated channel, and reduced channel bandwidth to account for the influence of Doppler spread. In contrast with other ad hoc networking use cases, VANET communication in general does not focus on unicast transmissions. Instead, the emphasis is put on the dissemination of information to all nodes located in a certain region. Typically, this functionality is achieved by combining media access control (MAC) level broadcasting with

\footnotetext{
*Correspondence: wim.vandenberghe@intec.ugent.be

1 iMinds, Department of Information Technology (INTEC), Ghent University, Gent, Belgium
}

Full list of author information is available at the end of the article geographic-aware forwarding schemes on the networking layer. In general, two different kinds of information messages are used: cooperative awareness messages (CAM) [2] and decentralized environmental notification messages (DENM) [3]. The former is utilized to continuously exchange status information such as location, heading, and speed with the other vehicles in the immediate environment. Hence, CAM beacons are restricted to singlehop broadcasts. The latter is applied when some specific piece of information regarding the environment, e.g., tail of traffic jam, slippery spot, obstacle on the road, etc. is to be communicated to all vehicles within a given area. Therefore, DENM messages are multi-hop broadcasted in the VANET.

An important challenge in the VANET domain is the scalability of data dissemination, which has been identified in numerous independent studies [4-11]. The goal of this paper is to realize a solution that overcomes this challenge, enabling scalable data dissemination in $\mathrm{V} 2 \mathrm{~V}$ and V2I communication. Note that next to the VANET scalability issue, there are also other VANET networking challenges to be overcome (e.g. IP session connectivity which relies on intelligent link-layer and IP-layer handovers as demonstrated by [12-14]), but these are out of scope

\section{黛 Springer}

(C) 2014 Vandenberghe et al.; licensee Springer. This is an Open Access article distributed under the terms of the Creative Commons Attribution License (http://creativecommons.org/licenses/by/2.0), which permits unrestricted use, distribution, and reproduction in any medium, provided the original work is properly credited. 
of the work presented in this paper. Here, we focus on the VANET scalability challenge for data dissemination: under dense traffic conditions, the large number of communicating vehicles can easily result in a congested wireless channel. As a result, delays and packet losses increase to a level where the VANET cannot be applied for safety applications anymore. In [4-11], the hidden node problem (Figure 1) is identified as one of the main drivers behind the performance decrease. The IEEE 802.11p MAC relies on a technique called carrier sense multiple access with collision avoidance (CSMA/CA) to coordinate transmissions on the wireless channel in a distributed manner. In short, a node can only transmit a packet if it has sensed that the wireless channel is idle for a sufficient amount of time. If it overhears the transmission of another node on the channel, it defers its transmission attempt until that transmission has finished. A hidden node $(\mathrm{H})$ however is not aware of a concurrent transmission because it is out of the range of the sender $(\mathrm{S})$ but in the range of the receiver (R). Therefore, it is possible that the hidden node will start sending its own data and interfere with the concurrent transmission between $\mathrm{S}$ and $\mathrm{R}$. To solve this problem, the technique of request-to-send (RTS) and clear-to-send (CTS) was developed. In this case, node S first broadcasts a short RTS message. The receiver node $\mathrm{R}$ replies with a CTS message, indicating the duration of the planned transmission. Because the hidden node is within reach of the receiver node, it will receive the CTS message and can derive the appropriate time to wait before attempting any transmission of itself. However, because there is no receiver defined in broadcast mode, IEEE 802.11 does not support RTS/CTS when broadcasting. As depicted in

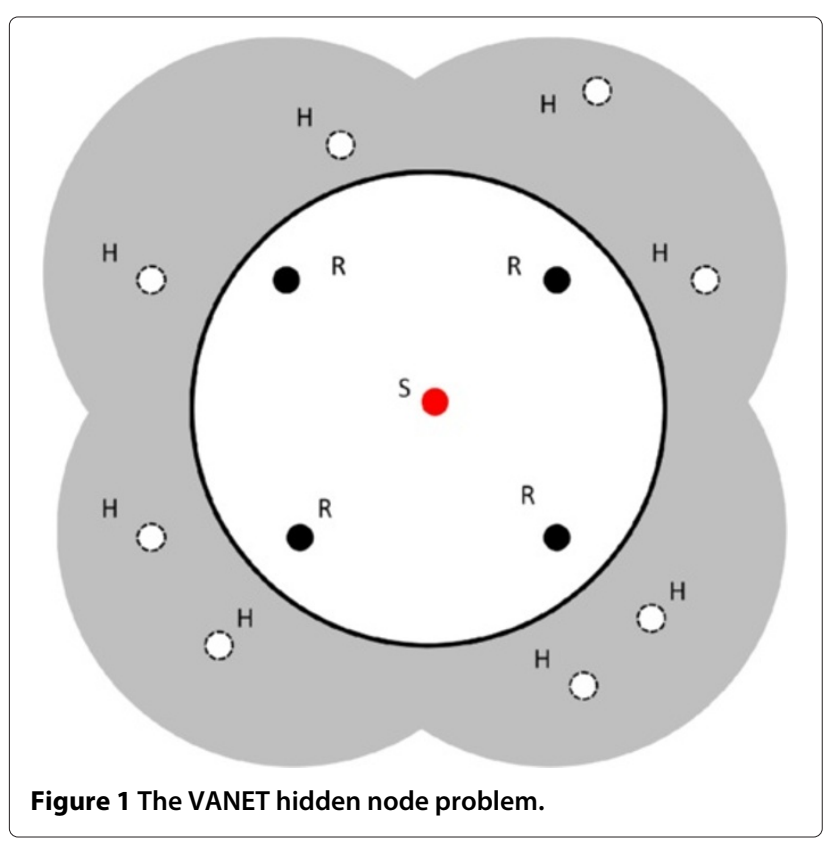

Figure 1, this leads to a high sensitivity of the VANET to the hidden node problem, since for a given node $\mathrm{S}$, a relatively large hidden node area (annotated grey) can be identified. This sensitivity is further increased by the fact that broadcasting also results in the ommision of MAC acknowledgment and retransmission techniques. Another cause of the VANET scalability problem is the consecutive freeze process (CFP) [9]. It refers to the situation where a station that has just completed a transmission chooses zero as its initial backoff time and hence starts transmitting immediately after a distributed coordination function (DCF) interframe space. This gives other stations no chances to back off. As discussed in [9], CFP occurs more frequently in broadcast situations, leading to high end-to-end delays. A final VANET challenge is the rapid variation of the channel at vehicular speeds. This results in increased packet loss when using a standard channel estimation scheme for IEEE 802.11p [15]. Hence, when targeting reliable VANET solutions, there is a need for solutions that enhance the packet success rate (PSR) at rising speeds while maintaining full compatibility with the IEEE 802.11p standard

Several techniques have been researched to tackle the VANET scalability problem. These are situated on all layers of the Open Systems Interconnection (OSI) protocol stack. In general, previous studies focused on applying a single technique, identifying the optimal parameter configuration and indicating the VANET performance gain. However, recent work has pointed out the need for a more holistic approach, where different techniques are combined to effectively solve the VANET scalability problem [16-18]. However, this is not as straightforward as it may seem, techniques that perform well independently might very well result in mutual compensations, ruling out each others performance gains. An example is the work of Baldessari et al. [19] which came across such difficulties when combining a transmit power and rate control algorithm.

In this paper, a holistic solution to the VANET scalability problem is established. We name this solution scalable data dissemination in vehicular ad hoc networks or SDDV. It is composed of several techniques spread across the different layers of the protocol stack. These techniques are characterized by a low level of complexity. This approach makes the solution more straightforward to implement, debug, and maintain. Simulation results are presented that illustrate the severity of the scalability problem when applying common state-of-the-art techniques (Section 3). Starting from this baseline solution, optimization techniques are gradually added to SDDV until the scalability problem is entirely solved. This requires the adoption of in total eight different techniques (Table 1) which are discussed in detail in Section 4. Section 5 determines the performance characteristics of the final SDDV 
Table 1 Summary of baseline VANET solution and the corresponding optimized techniques and parameters

\begin{tabular}{|c|c|c|c|c|c|}
\hline $\begin{array}{l}\text { SDDV } \\
\text { level }\end{array}$ & OSI layer & Baseline technique & Baseline parameters & SDDV technique & Final SDDV parameters \\
\hline 1 & Network & Simple flooding & & Opportunistic forwarding & $\begin{array}{l}S_{\max }=75, T_{\max }=75 \mathrm{~ms}, \\
a=2\end{array}$ \\
\hline 2 & Network & Simple flooding & & Irresponsible forwarding & Density based, $a=25$ \\
\hline 3 & MAC & Fixed transmission power & $33 \mathrm{dBm}$ & Fixed transmission power & $33 \mathrm{dBm}$ \\
\hline 4 & MAC & Fixed transmission rate & $6 \mathrm{Mbps}\left(\mathrm{QPSK} \frac{1}{2}\right)$ & Fixed transmission rate & 12 Mbps (16 QAM $\frac{1}{2}$ ) \\
\hline 5 & MAC & Fixed QoS class usage & $C A M=V O, D E N M=V O$ & Fixed QoS class usage & $\mathrm{CAM}=\mathrm{VO}, \mathrm{DENM}=\mathrm{VO}$ \\
\hline 6 & Applic. & Fixed beacon interval & $\begin{array}{l}\mathrm{CAM}=100 \mathrm{~ms}, \mathrm{DENM}=100 \\
\mathrm{~ms}\end{array}$ & Adaptive beaconing & $\begin{array}{l}\text { CAM }=\frac{130 \mathrm{~km} / \mathrm{h}}{\text { vehiclespeed }} \times \\
100 \mathrm{~ms}, \mathrm{DENM}=100 \mathrm{~ms}\end{array}$ \\
\hline 7 & $\mathrm{PHY}$ & $\begin{array}{l}\text { Standard channel } \\
\text { estimation }\end{array}$ & & $\begin{array}{l}\text { Decision feedback and } \\
\text { smoothing }\end{array}$ & $\begin{array}{l}\text { Single antenna, decision } \\
\text { feedback, and smoothing } \\
\text { over all carriers }\end{array}$ \\
\hline 8 & $\mathrm{PHY}$ & Standard packet capture & & $\begin{array}{l}\text { Preamble and data } \\
\text { switching }\end{array}$ & \\
\hline
\end{tabular}

Summary of baseline VANET solution derived from the current state of the art, and the corresponding optimized techniques and parameters included in SDDV.

configuration through extensive simulations. As final part of our research, Section 6 validates the simulation results by comparing the performance of the baseline solution and SDDV on the iMinds w-iLab.t wireless testbed [20].

\section{Methodology}

In this paper, an empirical approach is adopted. Performance analyses are based on simulation results, which are gathered using the wireless network simulator NS-2 [21]. A final validation of the solution is performed on real hardware using the iMinds w-iLabt.t wireless testbed. The applied performance metrics were chosen based on existing studies such as those introduced in Section 1. Examples of previously utilized metrics are probability of reception failure or its inverse form packet success rate (sometimes also called packet delivery probability), reachability within a given geographical area, saved rebroadcasts compared to simple flooding, and end-to-end delay. For this paper, it was chosen to focus on the end-to-end characteristics on the application level. The applied metrics are packet success rate and delay. These are analyzed both in the scope of CAM and DENM messages.

The simulation platform is based on NS-2. However, instead of applying the legacy implementation of the IEEE 802.11 physical (PHY) and MAC layers, the overhauled implementation of [22] is adopted. This leads to more representative simulations since this implementation introduces an accumulative interference model. This is a significant improvement compared to the legacy implementation and is of high importance when taking the hidden node problem into account. The simulated scenario resembles the one presented in [11] and is depicted in Figure 2. A stretch of highway is simulated with a total length of $5 \mathrm{~km}$. Three lanes are defined in each direction, on each lane, the same average inter-vehicle distance is configured for a single simulation. Considered inter-vehicle distances are 160, 80, 40, and $10 \mathrm{~m}$. This corresponds with traffic conditions during the night,

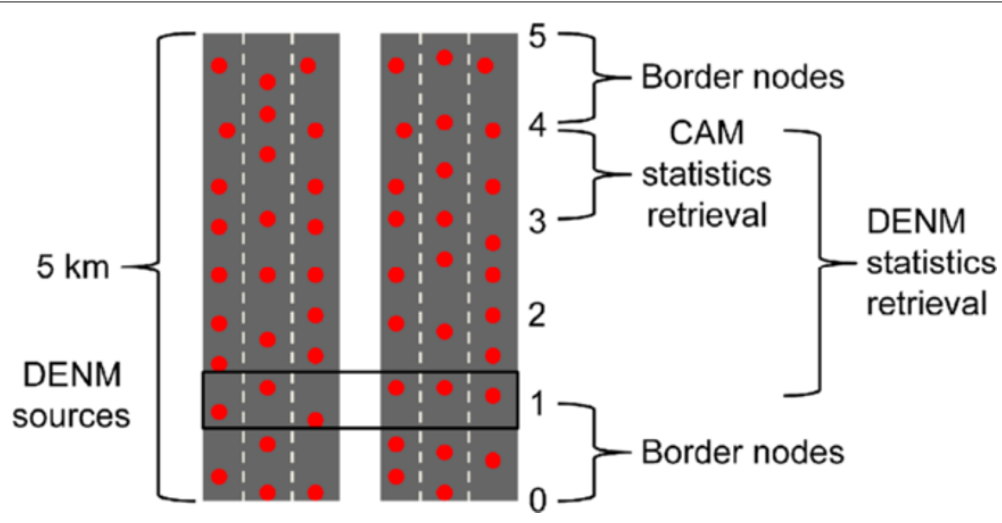

Figure 2 Applied simulator scenario. 
normal daytime traffic, intense but still flowing traffic, and jammed traffic. The total amount of active nodes in a single simulation is then $188,375,750$, and 3,000 vehicles. To exclude border effects, no performance statistics are gathered regarding the nodes located in the first and last kilometer of the simulated highway. CAM statistics are recorded for all nodes located between 3 and $4 \mathrm{~km}$ after the start of the highway stretch. DENM reception statistics are gathered for all nodes between 1 and $4 \mathrm{~km}$. A total of six active DENM sources are configured during the simulation (the first six nodes after the mark of 1 $\mathrm{km}$ ). It is chosen not to include actual node movement in the simulations. Instead, the simulation can be seen as a snapshot of the VANET configuration, in which the relative position of the nodes does not change at all during the simulation. This makes it more straightforward to interpret performance results since nodes do not continuously move in and out of each other's transmission range. To make sure that the wireless channel is loaded appropriately during performance measurements, the simulation is divided in three different phases. A single simulation run covers a simulation time of $35 \mathrm{~s}$, during which all nodes are transmitting CAM messages and the six DENM sources introduce both CAM and DENM messages in the network. Those DENM messages are forwarded by the other nodes using different techniques as described in Section 4.1. The first $10 \mathrm{~s}$ define the warm-up phase, during the next $20 \mathrm{~s}$, the performance measurements are recorded, and the last $5 \mathrm{~s}$ are used as a cool-down phase.

To model the effect of node velocity (including Doppler spread) on the link quality during these NS-2 simulations, link impairment is applied. This introduces an appropriate amount of random packet loss based on the chosen modulation, speed difference between sender and receiver, and the signal-to-noise (SNR) ratio of the received packet. This link impairment is based on a Matlab model that was developed to simulate the physical layer of the IEEE 802.11p standard in a high level of detail, including the effects of Doppler spread. More information regarding this impairment technique can be found in previous work [23]. To calculate the speed difference between nodes in NS-2, a fixed velocity value is configured for each node based on the lane in which it is located (see Table 2). To identify the nodes in our simulation results, they are numbered sequentially from start to finish, and each successive
ID corresponds with a node on the next lane. After the sixth lane, the numbering continues again at the first lane, selecting the next node within the lane. Hence, nodes 1 to 6 can be considered as the first row of nodes on the highway spread over lanes 1 to 6 , nodes 7 to 12 as the second row of nodes spread over lanes 1 to 6 , nodes 13 to 18 as the third row, and so on.

The applied VANET networking stack was implemented using the Click modular router framework. This is a modular software router platform originally developed by the Massachusetts Institute of Technology (MIT) with subsequent development by a broad research community [24]. This framework enables very efficient prototyping of networking protocols. It also supports the execution of the same implementation both within the NS-2 simulator and on real hardware. To guarantee that the performance measurements are performed equally during both kinds of experiments, the corresponding application level functionality was also implemented within the Click framework. More details regarding our specific VANET stack implementation are given in [25].

A final validation of the solution is performed on real hardware using the iMinds $\mathrm{w}$-iLabt.t wireless testbed. This is useful because simulations of wireless networks are not entirely representative for the real-life performance. This can be caused by several factors, as explained in our previous work [23]. We also refer to this publication for more information regarding the suitability of this testbed for VANET research. In short, the testbed cannot replace the simulator, but it is a useful tool to identify any possible issues that could arise in real-world deployments, but which are not adequately modeled by the simulator. One specific thing to mention here is that as described in [23], the lack of actual node movement in this testbed is compensated by the adoption of the same link impairment techniques as described above for the simulator.

\section{Performance evaluation of baseline solution}

Several techniques and parameter values are quite common in VANET literature. Together, they define the baseline VANET solution that is compliant with the current state of the art. In terms of forwarding strategy for multihop broadcasts, simple flooding is proposed by common standards such as CALM-FAST [26] and ETSI GeoNetworking [27]. In this paradigm, every node will relay

Table 2 Node velocities $(\mathrm{km} / \mathrm{h})$ based on the lane in which they are positioned

\begin{tabular}{|c|c|c|c|c|c|c|}
\hline Inter-vehicle distance & Lane 1 & Lane 2 & Lane 3 & Lane 4 & Lane 5 & Lane 6 \\
\hline $160 \mathrm{~m}$ & -100 & -115 & -130 & 130 & 115 & 100 \\
\hline $80 \mathrm{~m}$ & -100 & -115 & -130 & 130 & 115 & 100 \\
\hline $40 \mathrm{~m}$ & -100 & -110 & -120 & 120 & 110 & 100 \\
\hline $10 \mathrm{~m}$ & -25 & -25 & -25 & 25 & 25 & 25 \\
\hline
\end{tabular}


the received message if this node is located within the geographical destination area of the message and the hop-count limit has not been reached. In the ETSI standard, other forwarding strategies are briefly introduced; however, the standard annotates them as experimental and only obliges simple flooding. A common transmission power value is $2 \mathrm{~W}$ or $33 \mathrm{dBm}$, which corresponds with the maximum allowed equivalent isotropically radiated power (EIRP) for non-emergency vehicles in the IEEE 802.11p standard. As discussed in [28], in general, the default chosen data rate in VANETs is 6 Mbps. Because CAM and DENM messages are related to road safety applications, they are often annotated with the highest 802.11p QoS class, VO. The message generation frequency typically is $10 \mathrm{~Hz}$, since this is required by several of the cooperative road safety applications defined in $[29,30]$. As analyzed in previous work [23], the typical data size of both secure CAM and DENM messages is 300 bytes.

The performance characteristics of this baseline VANET approach are depicted in Figure 3 (CAM characteristics) and Figure 4 (DENM characteristics). The diagrams in the left column of Figure 3 indicate the CAM PSR values for the different envisaged vehicle densities, increasing from top to bottom. Every point on such a figure corresponds with the tuple (sender node ID, receiver node ID). The color of the point indicates the percentage of CAM broadcast messages originating from that sender that were actually received by that receiver. It varies between blue (no communication possible) and red (perfect communication). On the diagonal, the sender and receiver are the same. On this point, the value corresponds with the number of messages that the node actually created during the experiment. This way, it can easily be inspected if the requested data load was generated during the experiment. If so, the entire diagonal should be dark red. Using this color-coded approach, a convenient overview can be given of the specific communication characteristics of the entire simulated scenario. The average of all depicted values (except the control values on the diagonal) is also displayed on top of the figure. Although this average conceals many of the details given in the figure, it is a useful characteristic to compare the performance of different VANET optimization techniques. The illustrations in the right column of Figure 3 are very similar; they represent the average end-to-end delay for the CAM messages. In this case, the color scale varies between 0 and $100 \mathrm{~ms}$. This upper boundary was chosen because [30] defines $100 \mathrm{~ms}$ as the maximum allowed delay for most ITS applications. The color scheme of the delay charts is inverted compared to the PSR figures. This is because in case of PSR, a higher value is to be pursued, while in the case of delay, a low value is preferred. By inverting the color scheme, both types can more easily be compared: in both cases, the aim is to find VANET techniques that introduce as much red and as few blue points as possible.

The illustrations in Figure 4 focus on the DENM characteristics. Again, the vehicle density rises from top to bottom, and the left column presents the PSR while the right column focuses on delay. The $X$-axis of the charts corresponds with the receiver node ID, the $Y$-axis with the PSR or average delay value. Every series on the figure corresponds with a different sender node. So one series on the figure illustrates to which degree the messages created by a single source could be received by all other nodes and with which average delay. To avoid overloading the figure, only half of the DENM sources are depicted. In case of PSR, the $Y$-axis adopts a linear scale, while in case of delay, a logarithmic scale is applied. The average value is also depicted to allow easy comparison.

On Figures 3 and 4, the VANET scalability problem can clearly be observed. For increasing vehicle densities, the CAM PSR decreases significantly, while the delay under most intense traffic conditions become too large to support safety applications at all. Similar conclusions can be drawn regarding the DENM performance characteristics.

\section{Gradual composition of the holistic SDDV solution}

In this section, we present our holistic solution to the VANET scalability problem, SDDV. The starting point of our solution is an optimized approach to packet forwarding. Once this has been defined, other techniques spread across the different layers of the OSI networking stack are investigated. This allows us to gradually expand the SDDV suite of VANET techniques until a satisfactory combination is identified that effectively solves the VANET scalability problem. During this process, we will rely on extensive simulation efforts because the controlled environment of a wireless network simulator enables us to observe small differences in applied techniques and parameter values. Experiments on actual hardware are also included in this paper; for practical reasons, these kind of experiments were limited to the comparison of the completed SDDV solution with the baseline configuration described in Section 3.

\subsection{Optimizations on the networking layer}

As described in Section 3, simple flooding is a common approach to message forwarding in VANETs. However, from a wireless channel point of view, this techniques results in a significant waste of resources. As can be seen in Figure 5, every node within the transmission range of a sender node will forward a broadcasted message. However, the same geographical coverage of data dissemination could easily be achieved by selecting the relaying nodes more intelligently. Two common techniques to 


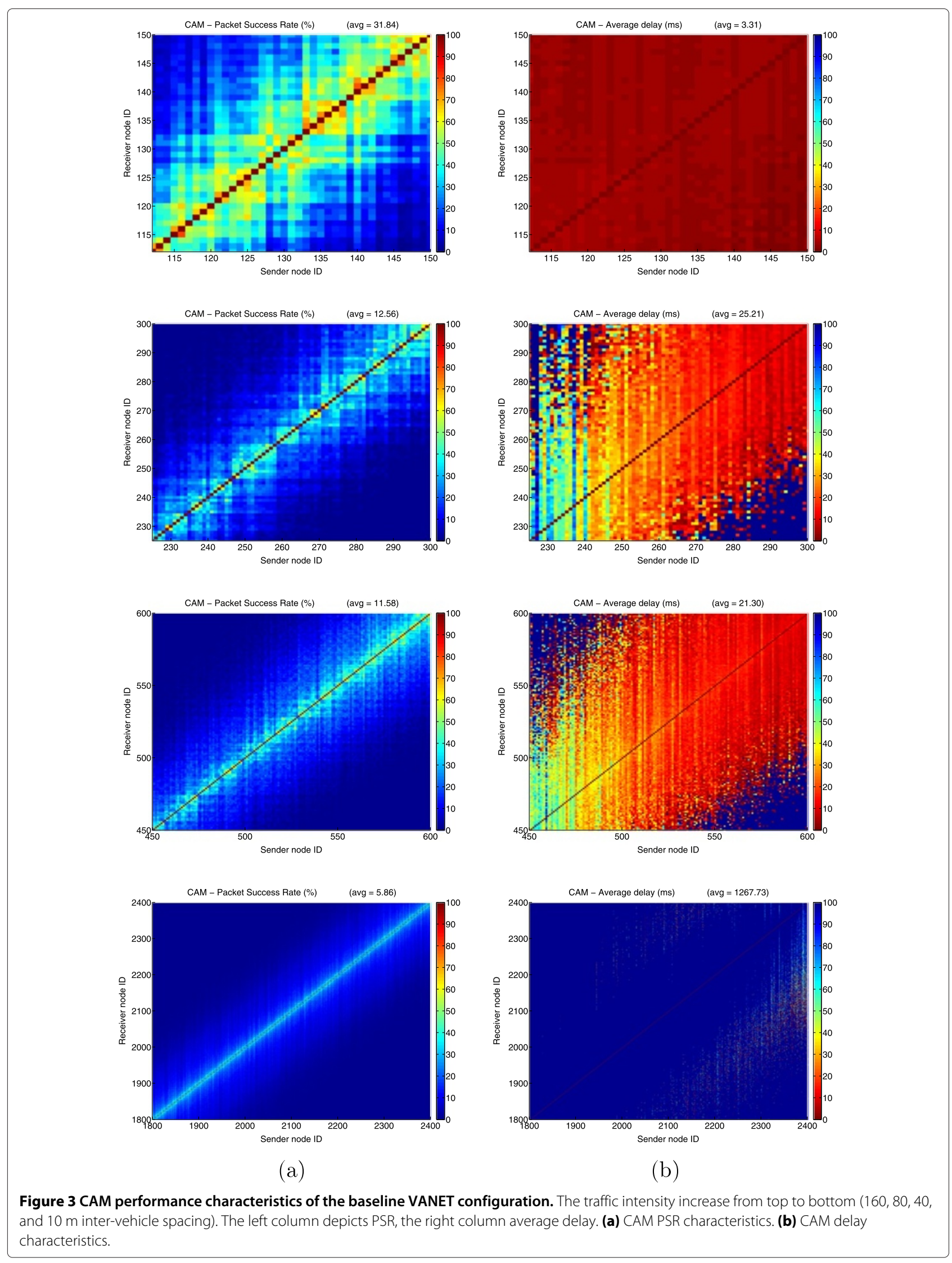



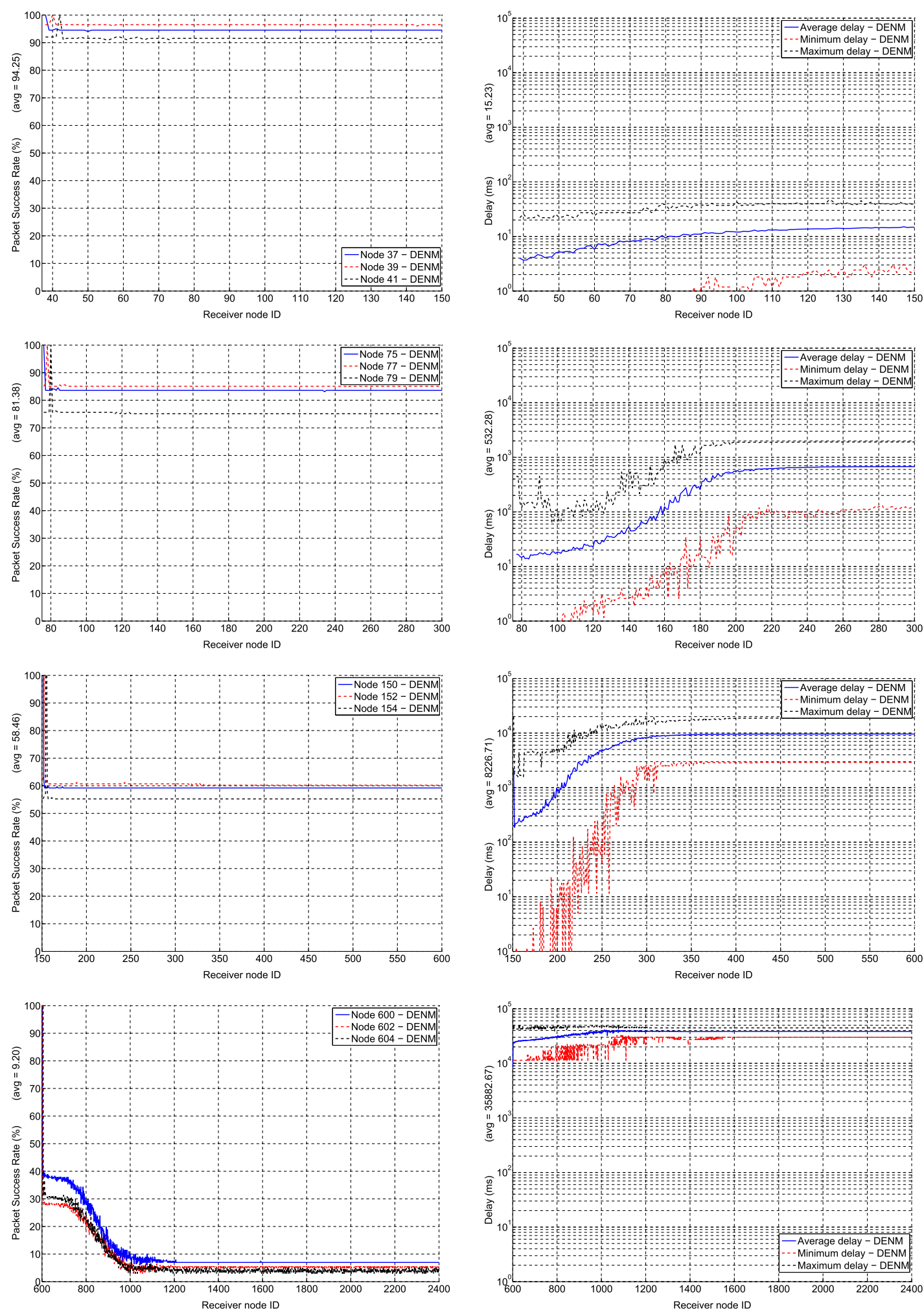

(a)

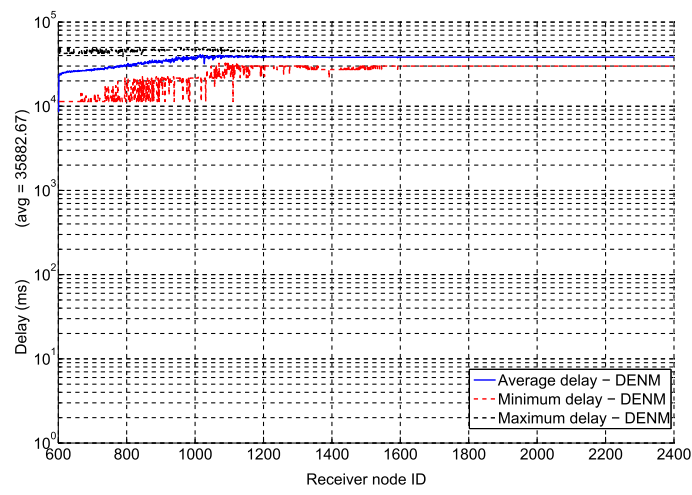

(b)

Figure 4 DENM performance characteristics of the baseline VANET configuration. The traffic intensity increase from top to bottom (160, 80 , 40, and $10 \mathrm{~m}$ inter-vehicle spacing). The left column depicts PSR, the right column average delay. (a) DENM PSR characteristics. (b) DENM delay characteristics. 


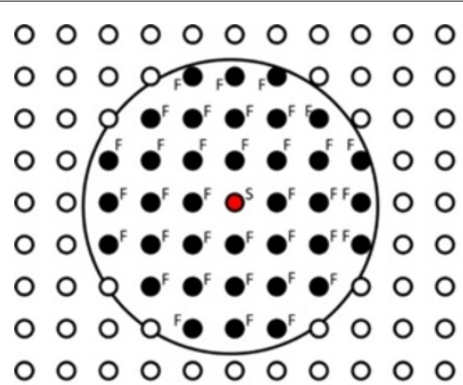

(a)

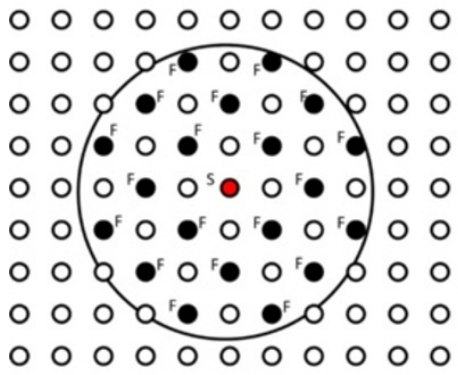

(c)

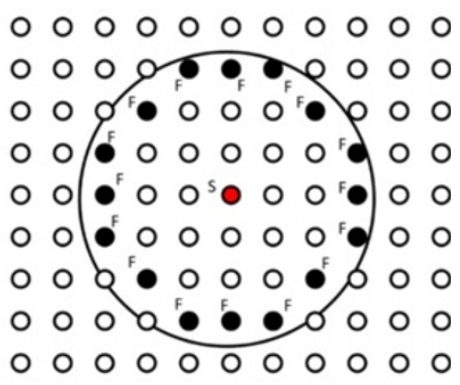

(b)

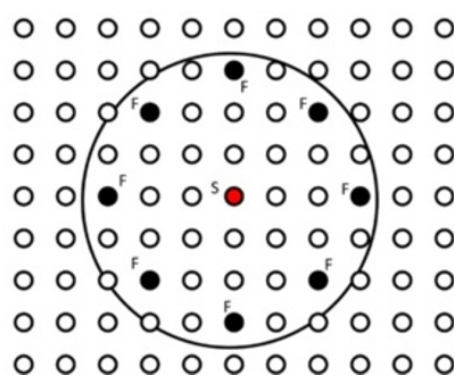

(d)

Figure 5 Illustration of the different message forwarding strategies applied in this paper. Node $\mathrm{S}$ is the sender node, and nodes $\mathrm{F}$ are the selected forwarding nodes. (a) Simple flooding. (b) Opportunistic forwarding. (c) Irresponsible forwarding. (d) Opportunistic + irreponsible forwarding.

reduce this level of forwarding redundancy are opportunistic forwarding and irresponsible forwarding. In the next two subsections, each of them is introduced and the performance gain is analyzed. We also study the combination of both techniques in the last subsection and show that a novel approach improves performance further.

\subsubsection{Opportunistic forwarding}

The basic idea behind opportunistic forwarding is that relay nodes are selected in such a way that the highest geographical gain is introduced per message retransmit. In other words, when a sender S broadcasts a DENM message, only the nodes located at the edge of S' transmission domain are selected as forwarding nodes F (Figure 5). To achieve this behavior, every node has to wait a certain time before it gets the opportunity to retransmit a received message. This waiting time is dependent on the distance between the previous sender of the message and the possible relay node: the larger the distance, the shorter the waiting time. If the waiting timer of a relay candidate expires and it has not yet overheard a retransmission of the same packet by any of its neighbors, then it is allowed to retransmit the message. However, when such a retransmit is overheard during the waiting phase, the node cancels its timer and destroys the message.

This technique was already investigated extensively for the VANET context [31-36]. All authors except [33] apply the actual distance between sender and relay node to determine the duration that the relay candidate has to wait. The function to calculate this backoff time is sometimes formulated differently but can always be converted to the following general wait time function for opportunistic broadcasting:

$$
T=T_{\max } \times\left(\frac{\left(\Delta d_{\max }\right)^{a}-(\Delta d)^{a}}{\left(\Delta d_{\max }\right)^{a}}\right)
$$

In this formula, $T$ is the calculated backoff time and $T_{\max }$ defines the maximum backoff time that is allowed. $\Delta d_{\max }$ is the maximum gain that can be reached in terms of the distance between the previous sender and the relay node, while $\Delta d$ is the actual gain in terms of distance if the node performing the calculation would become the relay node. Several values for the exponent $a$ can be found in literature. Various authors apply the value $1[31,35]$, the values $2[34,36]$, and 0.5 [34] that have also been explored before. Two other parameter values are also of importance in (1): $T_{\max }$ and $\Delta d_{\max }$. The former can be varied in order to obtain maximum network performance. The latter has an importance influence on the results of (1), but it is hard to estimate a suitable value for this parameter. It corresponds with the maximum transmission range of the sender, which can fluctuate based on environmental circumstances such as antenna quality and position on the vehicle, external interference, landscape elements that 
influence fading or multi-path reflections, etc. Therefore, the approach of [33] was adopted in SDDV: the backoff duration for opportunistic forwarding is calculated based on the signal-to-noise (SNR) value of the received message and not the distance between the sender and the relay candidate. The wait time function in this case becomes:

$$
T=T_{\max } \times\left(1-\frac{\left(S_{\max }\right)^{a}-(S)^{a}}{\left(S_{\max }\right)^{a}}\right)
$$

In this formula, $S_{\max }$ is the maximum SNR value that is possible when receiving a message. $S$ is the actual SNR value of the received message that the calculating node might relay. The suitable value for $S_{\max }$ can easily be determined. Taking into account IEEE 802.11p characteristics such as a maximum EIRP of $2 \mathrm{~W}$, a frequency of $5.9 \mathrm{GHz}$ and a noise floor of $-99 \mathrm{dBm}$, together with the observation that vehicles on a highway are spaced at least $4 \mathrm{~m}$ apart, results in a maximum SNR value of $75 \mathrm{~dB}$ when taking the free space path loss into account. Another advantage of using SNR instead of distance is the fact that SNR values are always available, even under conditions with distorted GPS reception such as the urban canyon, tunnels, etc.

With a fixed $S_{\max }$ value of $75 \mathrm{~dB}$, two parameters are left to optimize: $T_{\max }$ and $a$. In our research, a large number of combinations was explored in the simulator, with $T_{\max }$ varying between 1 and $500 \mathrm{~ms}$, and $a$ taking one of the values $0.5,1$, or 2 . During those tests, the focus was put on the improvement of CAM PSR. Most profit could be achieved when using a $T_{\max }$ value of $200 \mathrm{~ms}$ and an $a$ value of 1 . The corresponding CAM PSR values are given in the left part of Figure 6. It can be observed that a significant gain is achieved compared to the simple flooding technique. However, when focusing on the average DENM delay depicted in the right-hand side of the figure, it can be observed that such a high CAM PSR introduces relatively large DENM delays. This is because for a rather high $T_{\max }$ value of $200 \mathrm{~ms}$, quite a lot of time is lost waiting in the backoff phase of the opportunistic routing approach. We therefore refer to this configuration as the aggressive approach. By further investigating our experimental results, it was possible to define a more balanced parameter set. For a $T_{\max }$ of $75 \mathrm{~ms}$ and a $a$ value of 2, the gain in CAM PSR is a bit lower than in the aggressive approach, but it remains significant compared to the simple flooding approach. On the other hand, the DENM delay values are remarkably lower in the balanced than in the aggressive approach. This trade-off between CAM PSR and DENM delay is illustrated in Figure 6 together with the corresponding impact of both the aggressive and balanced approach.

\subsubsection{Irresponsible forwarding}

The main concept of irresponsible forwarding is that when a node receives a multi-hop broadcast packet, it will retransmit that packet with a specific probability (Figure 5). Two approaches to define this probability can be found in literature. References $[6,37,38]$ make the probability dependent of the distance between the previous sender and the relay candidate. In $[34,39,40]$, the probability is determined based on the neighborhood density. Both approaches were experimentally validated in the scope of this paper.

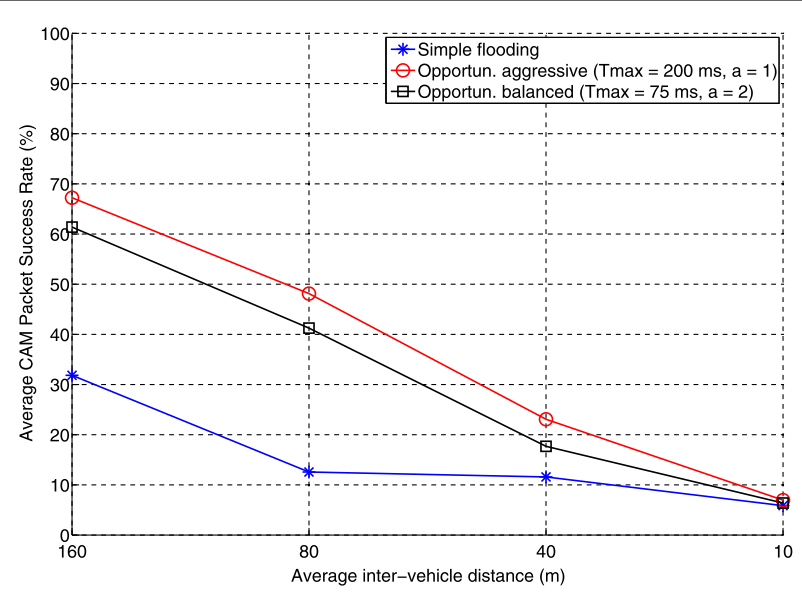

(a)

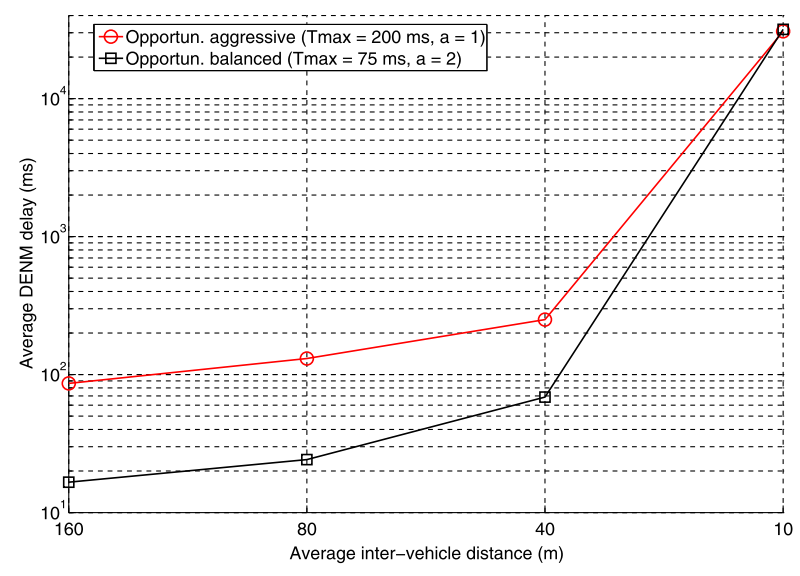

(b)

Figure 6 Effect of opportunistic forwarding on the VANET performance. In terms of CAM PSR, best results are achieved for a $T_{\text {max }}$ value of 200 $\mathrm{ms}$ and $a$ value of 1 . However, this results in a relatively large DENM delay. This configuration is called the aggressive approach. When configuring a $T_{\max }$ value of $75 \mathrm{~ms}$ and an a value of 2, CAM PSR profit is still significant, and the DENM delay is clearly lower. This is called the balanced approach. (a) CAM PSR values for opportunistic forwarding. (b) DENM delay values for opportunistic forwarding. 
In case of a probability based on the distance between the sender and possible forwarder, again, a function based on SNR values was applied in our research. In that case, the probability $p$ that the receiver node will retransmit the message is as follows:

$$
p=\frac{\left(S_{\max }\right)^{a}-(S)^{a}}{\left(S_{\max }\right)^{a}}
$$

In this formula, $S_{\max }$ is again the maximum SNR value that is possible when receiving a message, and $S$ is the actual SNR value of the received message that the calculating node might relay. In this case, the only parameter to evaluate is $a$. The values $0.5,1$, and 2 were simulated. Best results were obtained for a value of 0.5 . However, even for this configuration, the balanced opportunistic forwarding scheme clearly performed better than the irresponsible one. Therefore, the focus was shifted towards another approach, where the probability $p$ that the receiver node will retransmit the message is now based on its neighborhood density. In that case, $p$ is calculated as follows:

$$
p=\frac{a}{N}
$$

In this formula, $N$ is the amount of node neighbors that can be reached with a direct one-hop transmission. The parameter $a$ has no physical meaning. It is the only parameter that can be varied. In our experiments, this parameter was gradually varied between 1 and 35 in steps of 5 . The highest CAM PSR results were observed for rather low (aggressive) values such as 1 or 10 (see Figure 7). In this case, only a small amount of nodes will rebroadcast a specific DENM message. This leads to a lower load on the wireless channel, giving more room for CAM activities. The downside however is that a trade-off could be identified between CAM PSR and DENM PSR: low values for the $a$ parameter result in high CAM PSR values but low corresponding DENM PSR values (Figure 7). This illustrates that not enough nodes are selected to forward DENM messages, leading to reduced end-to-end DENM performance. Therefore, similar to the approach taken in Section 4.1.1, this aggressive approach should be replaced by a more balanced configuration. Our experiments revealed that in that balanced case, the corresponding value of the parameter $a$ is 25. As depicted in Figure 7, this results in a good equilibrium between CAM and DENM PSR.

\subsubsection{Combined approach}

In the previous sections, it was demonstrated that both opportunistic and irresponsible forwarding can significantly increase network performance compared to the simple flooding technique. However, for each technique, there is a trade-off between CAM PSR improvement and DENM performance decrease: delay in case of opportunistic forwarding, PSR in case of irresponsible forwarding. This brings up the question if both techniques can be combined in such a way that the performance gain is larger than in the case of a single technique, without jeopardizing DENM characteristics.

Such a combination has been introduced before [34,41]. However, in both cases, the adopted approach differed from the one taken in SDDV. The p-IVG protocol presented in [34] applies opportunistic forwarding as the

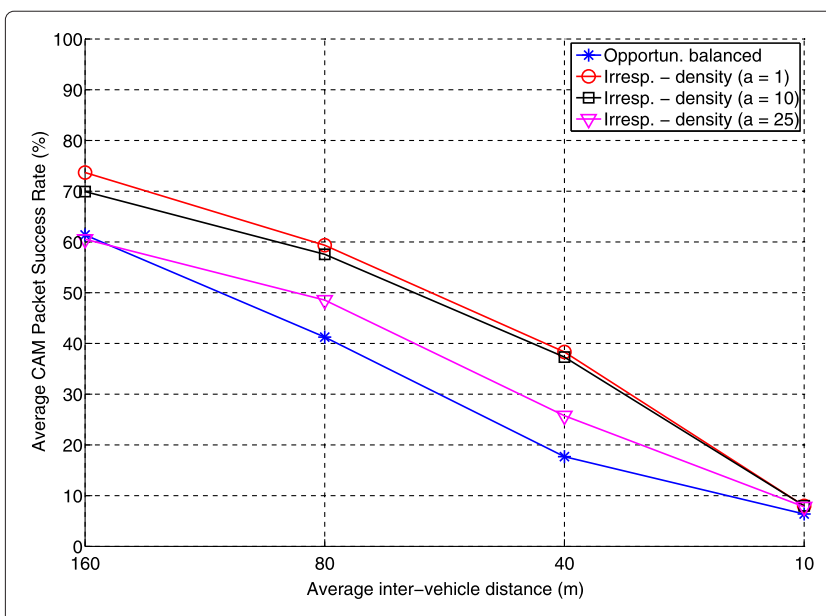

(a)

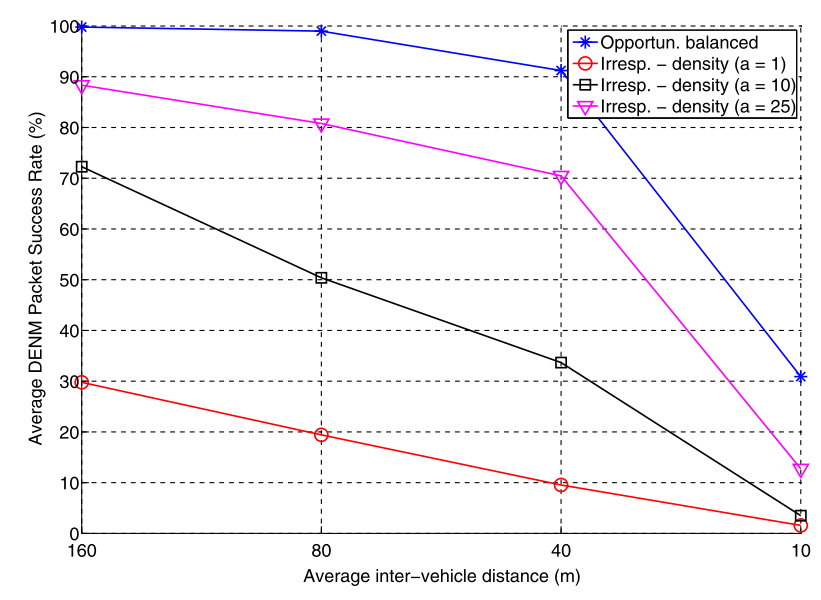

(b)

Figure 7 Effect of irresponsible forwarding on VANET performance. In terms of CAM PSR, best results are achieved for a low a value (1 or 10). However, this results in low DENM PSR values. When configuring an a value of 25, CAM PSR profit is still significant, and the DENM PSR is clearly affected less. This configuration is the balanced approach to irresponsible forwarding. (a) CAM PSR values for irresponsible forwarding. (b) DENM PSR values for irresponsible forwarding. 
base technique and then relies on irresponsible forwarding to further reduce the amount of relay nodes. However, the decision function of their irresponsible forwarding technique uses a value of 1 for the parameter $a$. As discussed in Section 4.1.2, this value is too aggressive, since it can lead to a significant decrease of DENM PSR values. The study of Oh et al. [41] on the other hand also combines opportunistic and irresponsible forwarding, but the decision function of the irresponsible forwarding component is not based on neighborhood density. Instead, it relies on the inter-arrival time of VANET packets. However, we believe that this is a less suitable metric, since it only measures the congestion on the wireless medium and provides no information regarding the amount of suitable relay candidates. This metric cannot distinguish a scenario with a large number of beaconing vehicles from a scenario with just a few vehicles which are consuming a large amount of wireless resources.
In our simulations, both the aggressive and balanced configurations of opportunistic and irresponsible forwarding were combined. The results are depicted in Figure 8. It should be mentioned that the sequence in which these techniques are applied is the same as in pIVG: first opportunistic forwarding is executed, then the remaining set of relay candidates is further reduced using the irresponsible forwarding technique. The inverse order was also simulated but led to inferior results. It can be observed that, as expected, the combination of the aggressive approaches results in an unacceptable decrease of DENM PSR values and an increase of DENM delay. The combination of the balanced approaches on the other hand is promising. Compared to the previous results regarding the application of only opportunistic or only irresponsible forwarding, all VANET performance characteristics are further improved without introducing any negative side effects. Therefore, SDDV adopts this specific
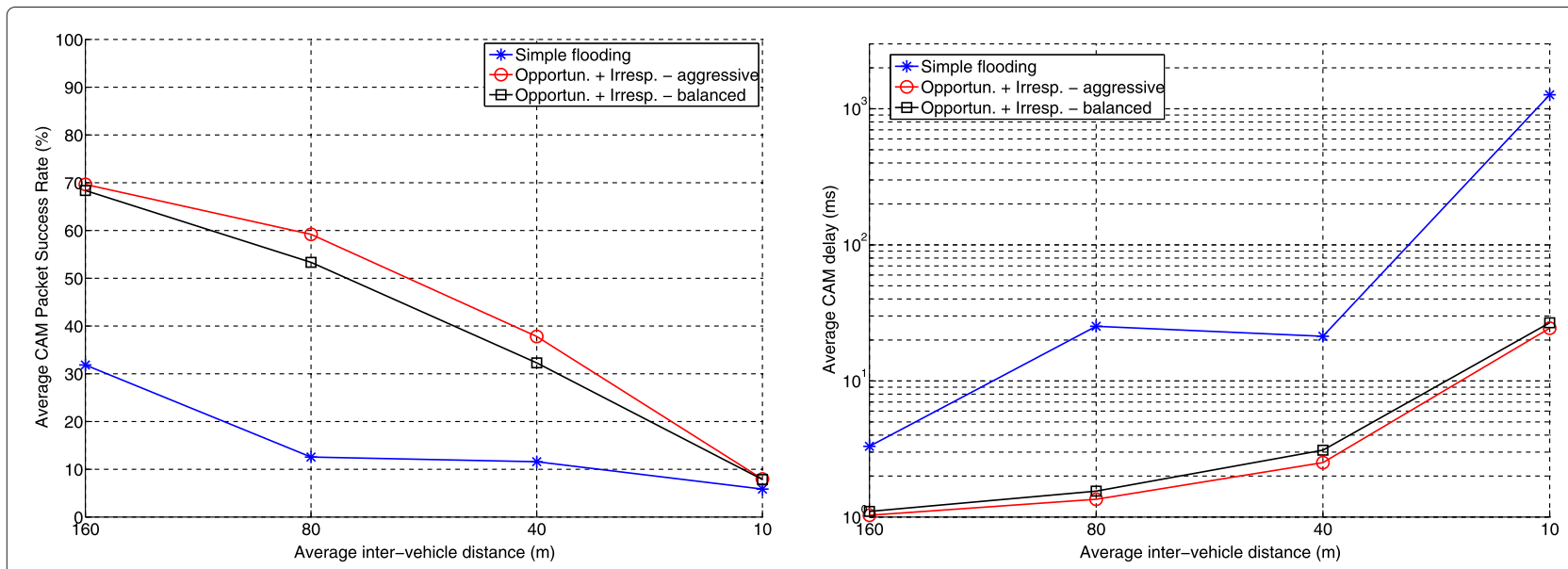

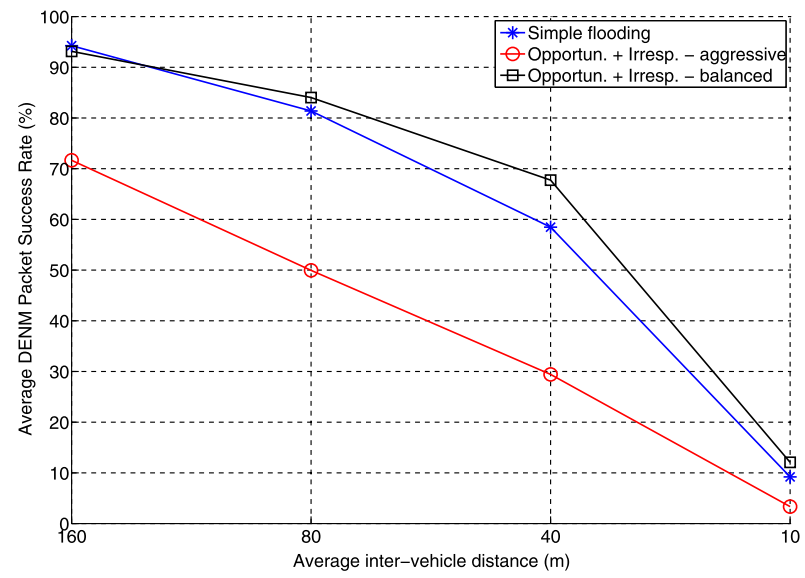

(a)

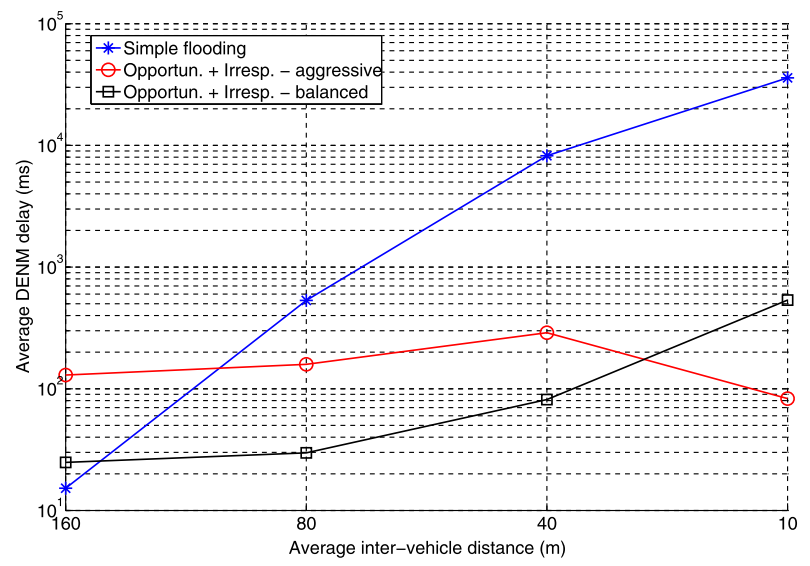

(b)

Figure 8 Performance characteristics of the combination of opportunistic and irresponsible forwarding. The top row presents CAM characteristics, and the bottom row DENM characteristics. Combining the aggressive approaches results in an unacceptable decrease of DENM performance (low PSR, high delay). However, combining the balanced approaches leads to better performance compared to both individual techniques, without introducing any negative side effects on DENM performance. (a) Packet success rate. (b) Delay. 
combination of opportunistic and irresponsible forwarding as the default approach to packet forwarding.

\subsection{Transmit power control}

Now that the networking layer optimizations of the SDDV protocol are defined, it can be explored if the inclusion of other techniques results in additional performance gains. Transmit power control is one of the common techniques in the context of the VANET scalability problem. The basic idea is that the load on the wireless channel is reduced by lowering the transmit power of the nodes in case of high vehicle density. In case of low vehicle density, higher transmit powers are applied to ensure VANET connectivity. A good example of such a solution is the work of Torrent-Moreno et al. [42], which introduces the DFPAV algorithm. This algorithm dynamically adjusts the transmit power of CAM beacons to guarantee that a specific threshold called the maximum beaconing load (MBL) is never exceeded. DENM messages on the other hand are always transmitted at the maximum possible transmit power. To derive appropriate power values for the CAM beacons, the D-FPAV algorithm relies on the exchange of specific information between VANET nodes. This leads to additional communication overhead. This overhead has been reduced in the work of Mittag et al. [43], which presented SPAV, a segment-based evolution of D-FPAV that relies on a distributed vehicle density estimation protocol called DVDE.

Alternative techniques exist that do not exchange any control data to perform transmit power control. Instead, each node dynamically adjusts its transmit power based on the measured value of a specific network metric. Two different metrics are proposed: neighborhood density $[44,45]$ and channel busy time $[19,46]$.

Before implementing our own approach to transmit power control, some exploratory experiments were performed. In every experiment, the current level of SDDV was simulated (level 2). During a single simulation run, the transmit power was kept static, different simulations were executed with values gradually decreasing from 33 to $3 \mathrm{dBm}$ (in steps of $5 \mathrm{dBm}$ ). For each transmit power value, the four different traffic intensities were simulated. The results indicated that from the CAM PSR perspective, there is no need to dynamically adjust the transmit power: the maximum value of $33 \mathrm{dBm}$ corresponded with the best performance for all four considered traffic intensities. When focusing on the DENM PSR, however, it is beneficial to lower the transmit power for the highest vehicle density (10 $\mathrm{m}$ inter-vehicle distance). In the other cases, no distinct profit could be observed.

The next step was to implement an actual power control solution. Because this paper aims to combine techniques with a relatively low level of complexity, it was chosen not to adopt the D-FPAV or SPAV algorithms. Instead, we adopted the approach of dynamically adjusting the transmit power of each node based on its measured neighborhood density. In our solution, both CAM and DENM messages are transmitted with the same transmit power since we consider both types of safety messages as equally important. Every second, the neighborhood density is compared to a threshold value $N_{\max }$. If the amount of single-hop neighbors is lower than this threshold, the transmit power is increased with $1 \mathrm{dBm}$, if it is higher, it is decreased with $1 \mathrm{dBm}$. The threshold value is calculated as follows:

$$
N_{\max }=\frac{a \times C}{S \times f}
$$

In this formula, the term $C$ indicates the available capacity on the wireless channel in terms of bits per second that can be communicated. This corresponds with 6 Mbps. $S$ represents the size of the transmitted messages. As mentioned in Section 3, this is 300 bytes for both CAM and DENM messages. The term $f$ corresponds with the frequency of creating and transmitting subsequent CAM and DENM messages. As discussed in Section 3, this is $10 \mathrm{~Hz}$. The parameter $a$ has no physical meaning, it allows to introduce a safety margin in terms of channel congestion. For instance, when setting this parameter to 0.8 , the power control algorithm will try to reduce the number of neighbors as soon as they together consume $80 \%$ of the channel capacity.

For the highest vehicle density, this approach was able to introduce an increase of the average DENM PSR value from $12 \%$ to $30 \%$. However, it was observed that the introduced power control distorts the uniform nature of the VANET performance. For some nodes, transmitted CAM messages were received by almost all nodes in the evaluated geographical zone, while for other nodes, transmitted CAM messages were only received by their closest neighbors. This phenomenon is caused by the fact that there is no coordination between the power control algorithms active on the different nodes. Therefore, we extended our approach with synchronization based on the GPS time. However, as depicted in Figure 9, in this configuration, the distortion in the uniform network performance (compared to the CAM PSR plots depicted in Figure 3) is still present.

It was decided not to include any form of transmit power control in SDDV because (1) the exchange of power control data is in contradiction with the goal of SDDV to include only relatively simple techniques, (2) because the approach based on neighborhood density thresholds introduces distortions in the uniform nature of VANET performance, and (3) because our initial experiments indicated that no huge profits can be expected when introducing transmit power control on top of opportunistic and irresponsible forwarding. Instead, a fixed transmit power 


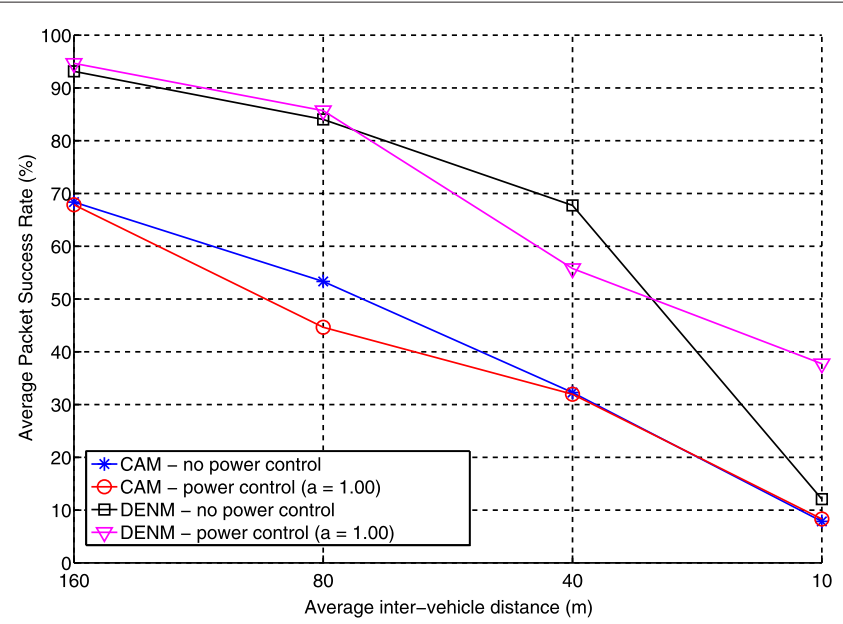

(a)

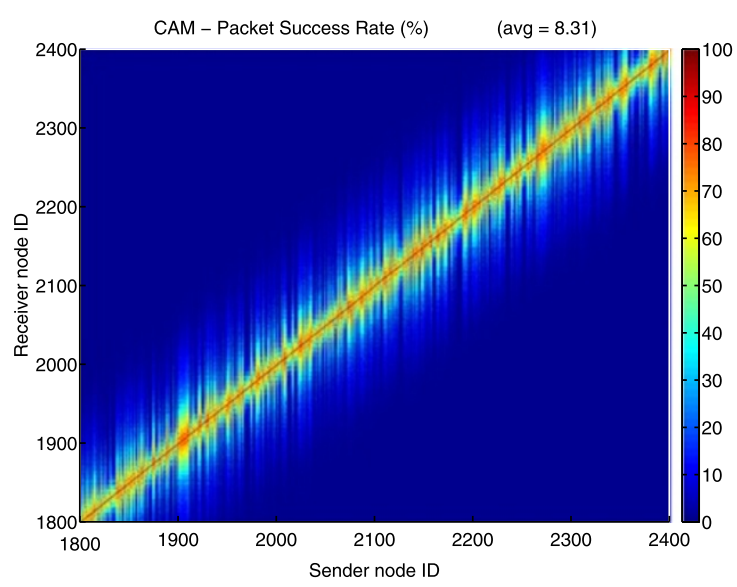

(b)

Figure 9 Results of the proposed power control algorithm using time synchronization. The only gained profit is a rise in DENM PSR for the highest traffic intensity (10 $\mathrm{m}$ inter-vehicle distance). However, CAM PSR at this density is not improved at all, and a distortion of the performance uniformity is introduced. (a) PSR overview of power control with GPS time synchronization. (b) Distortion of perfomance uniformity (with GPS time synchronization).

of $33 \mathrm{dBm}$ is defined as the next key characteristic of SDDV (level 3).

\subsection{Transmit rate control}

Similar to power rate control, the technique of transmit rate control is sometimes presented as a possible means to overcome the VANET scalability problem. When applying a higher transmit rate in the VANET, the capacity of the wireless communication channel is increased, which could (partly) resolve the channel congestion under highdensity traffic circumstances. However, the downside of increasing the transmit rate is that at the receiver side, the SNR value of the message has to be higher for successful packet reception. Hence, the communication range of the nodes is decreased. At higher rates, the wireless link is also more sensitive to node mobility (see Section 4.6) since in that case, the PHY layer applies more sensitive modulation techniques and less redundant levels of error correcting coding. These effects can negatively influence VANET end-to-end performance. Identifying the appropriate balance in this rate control trade-off is a challenge.

The work of Shankar et al. [47] and Wang et al. [48] proposed transmit rate control techniques to improve the quality of unicast communication links in the VANET. The former optimized the achievable goodput over a specific unicast link, while the latter achieved maximal energy efficiency. Because the focus of SDDV is on broadcasted data dissemination, these techniques are of less relevance to our solution. More closely related work is [28] which explained that the rate of $6 \mathrm{Mbps}$ is generally considered as the default value in VANET literature, though that this assumption is not rooted on strong technical considerations. Based on a large number of simulations, it concludes that also from a technical point of view $6 \mathrm{Mbps}$ is the optimal data rate. However, relatively small transmit power values were used (maximum range is $500 \mathrm{~m}$ ), not all nodes apply the same transmit rate during the experiment, and only CAM messages are taken into account. Ma et al. also investigated a scenario with only CAM messages; their results indicate that optimal results can be achieved with with 6 to $12 \mathrm{Mbps}$ in the IEEE 802.11p standard. The study of $\mathrm{Xu}$ et al. [49] on the other hand investigates the optimal data rate for different message repetition schemes on the MAC layer. They conclude that, depending on the chosen repetition algorithm, the optimal data rate varies between 6 and 12 Mbps.

Similar to our approach taken in Section 4.2 (power control), some experiments were performed to assess if expanding SDDV with techniques to dynamically adjust the transmit rate would result in worthwhile performance improvements. SDDV level 3 was simulated for all envisaged traffic intensities with a fixed transmit rate of 3 , 6 , and 12 Mbps. These rates were selected for experimentation because they provide the most robust error correction coding $\left(\frac{1}{2}\right)$ for the desired modulations available on the PHY layer (binary phase-shift keying or BPSK, quadrature phase-shift keying or QPSK and the variant of quadrature amplitude modulation 16-QAM). Note that 64-QAM was not considered because it is regarded in literature as too sensitive to be applicable in vehicular environments. The results of the experiments are given in Figure 10. It can be observed that $3 \mathrm{Mbps}$ clearly results in the worst PSR performance both in terms of CAM and DENM messaging. The 6 and 12 Mbps rates perform more 


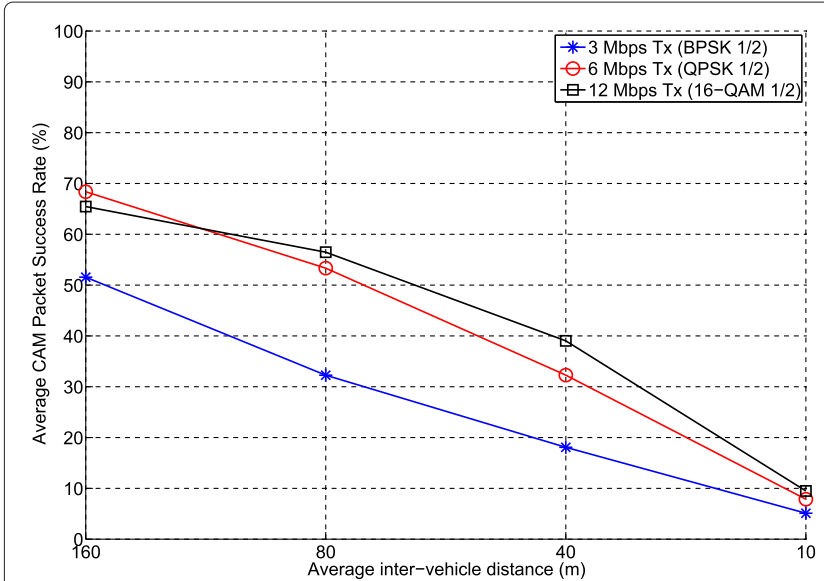

(a)

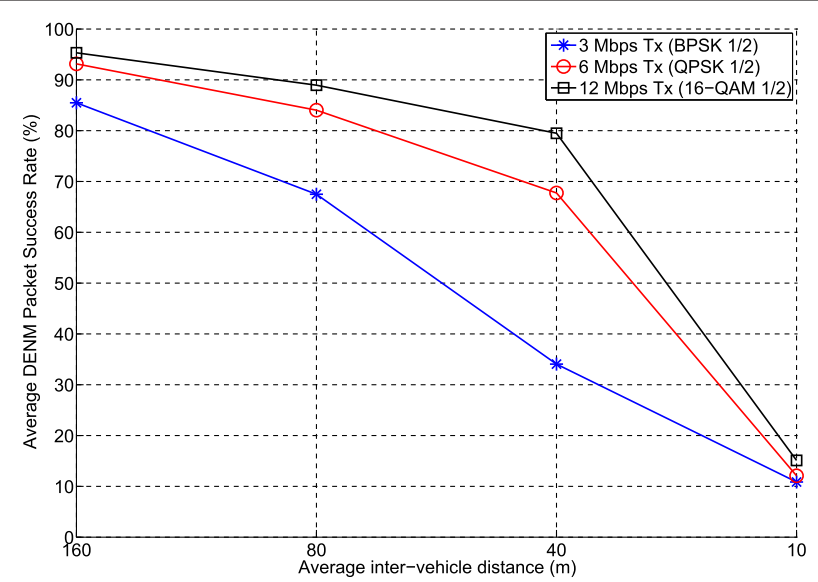

(b)

Figure 10 Effect of different static transmit rate values on network performance. It is obvious that under all circumstances, $12 \mathrm{Mbps}$ is the optimal choice. The only exception is the CAM PSR value for $160 \mathrm{~m}$; however, in this case, the difference is negligible. (a) Average CAM PSR for different fixed transmit rates. (b) Average DENM PSR for different fixed transmit rates.

closely to each other. Still the best performance is achieved when using the $12 \mathrm{Mbps}$ rate. Only for the lowest traffic intensity, the average CAM PSR value was slightly better in case of $6 \mathrm{Mbps}$. However, in that specific case, the difference between 6 and $12 \mathrm{Mbps}$ is negligible. It can be concluded that under all circumstances, the best results are achieved using the $12 \mathrm{Mbps}$ rate. Hence, it was decided not to include any form of transmit rate control in SDDV but to define a fixed transmit rate of $12 \mathrm{Mbps}$ as the next key characteristic of SDDV (level 4).

A point to mention is that during the experimentations described above and in Section 4.2 (power control), only one parameter was varied at a time, or transmit power or transmit rate. Since SDDV level 4 defines a fixed combination of $33 \mathrm{dBm}$ transmit power and $12 \mathrm{Mbps}$ transmit rate, it could be useful to further investigate if other fixed combinations of rate and power lead to even better results. Therefore, additional experiments were performed in order to cover all possible combinations of the envisaged transmit powers (33 to $3 \mathrm{dBm})$ and rates $(3,6,12$ Mbps). Careful evaluation of the results indicated that no other combinations achieved a better performance than the combination of $33 \mathrm{dBm}$ and $12 \mathrm{Mbps}$.

\subsection{Optimal selection of QoS class}

The IEEE 802.11p MAC layer is similar to the IEEE 802.11e enhanced distributed channel access (EDCA) extension that supports quality of service (QoS) [50]. This scheme resembles the CSMA/CA scheme of standard IEEE 802.11 called distributed coordination function or DCF, but it can differentiate between four different application categories: background traffic (BK), best effort traffic (BE), video traffic (VI), and voice traffic (VO). Different contention window $(\mathrm{CW})$ and arbitration inter frame space (AIFS) values are chosen for the different application categories, where VO has the highest priority and $\mathrm{BK}$ the lowest. For more information regarding the functionality of EDCA and the IEEE 802.11p MAC, we refer to our previous work [51].

Until now, the QoS class VO was always applied for the broadcasting of CAM and DENM messages. This is a common approach in literature, since both types of messages are safety related and VO is the highest QoS class. However, IEEE 802.11 defines that broadcasted messages should always be sent with a contention window value equal to $\mathrm{CW}_{\min }$. In the highest QoS classes, the values for $\mathrm{CW}_{\min }$ and AIFSN are small. This leads to small waiting times during contention, with a small end-to-end delay as a result. This is important for voice and video multimedia applications. However, the downside of this approach is that the probability increases that two contending nodes will choose the same time slot to start their transmissions, resulting in packet loss. In the context of the VANET scalability problem, it would therefore be useful to investigate if the usage of the lower QoS classes could lead to better networking performance.

A few studies could be found that elaborated further on this idea. In [52], it is investigated how the communication performance of a single node can be improved by assigning it to a higher QoS class than all the other nodes. This is not applicable for SDDV since we are looking for techniques that improve the performance equally for all VANET nodes. The research of [53] pursued this goal, experimenting with two techniques to improve general 
VANET performance. In the first one, all VANET nodes apply the same QoS class for all their messages, and this QoS class is varied over different experiments. The second technique adjusts the QoS class on a per-packet basis, using the success rate of previous packet transmissions as the dominant decision parameter. However, the authors only managed to achieve minor performance increases. Rebai et al. [54] defined sub-classes within the four existing QoS classes. Based on the success rate of previous transmissions, nodes calculate the appropriate sub-class for their messages. However, since broadcasting does not provide an acknowledgment mechanism on the MAC layer, this technique cannot be applied in our envisaged scenario. In the work of Adler et al. [55], nodes recalculate the appropriate value for their active contention timers after each DCF interframe space (DIFS), instead of using the value that was stored when the medium was sensed busy before. However, when only focusing on the presented PSR gains of this technique, the results are not as significant as desired for SDDV. In [56], the $\mathrm{CW}_{\min }$ value was varied based on traffic density. However, the presented simulations only included up to 20 vehicles, which does not provide sufficient information to draw conclusions regarding the impact of the technique on the VANET scalability problem.

Since the proposed techniques based on previous success rates cannot be used in broadcasting, and we expect similar levels of usefulness for all CAM and DENM messages, it was decided not to further investigate any of the dynamic per-packet based approaches. Instead, our focus was put on the identification of the optimal fixed combination of CAM and DENM QoS classes. If required, a dynamic alteration between different configurations based on a periodical evaluation of the neighborhood density would also be considered. Two different kinds of experiments were performed to explore the effect of different QoS class assignments. In the first series, both CAM and DENM messages were transmitted within the same QoS class. All four available QoS classes have been evaluated. The results demonstrated that when applying the same QoS class for both CAM and DENM messages, the default value VO turns out to be the optimal one.

In the second series of experiments, all combinations of QoS class assignments were simulated for which the priority of DENM traffic is higher than that of CAM traffic. The CAM results fell within the line of expectations: when CAM messages are transmitted in a lower QoS class, their PSR decreases and their delay increases. However, a peculiar behavior was observed regarding the DENM performance: the DENM PSR was influenced very positively by the new QoS strategy, but unexpectedly, the delay also rose quite significantly. This effect is related to SDDV's adoption of irresponsible forwarding based on neighborhood density. This density estimation is based on received CAM beacons. However, by deteriorating the CAM performance, a snowball effect is initiated: the forwarder starts to under-estimate the neighborhood density and, therefore, forwards more DENM messages. This increases the load on the wireless channel; as a consequence, the CAM performance further decreases, the irresponsible forwarder under-estimates the neighborhood density even more, forwards even more DENM messages, further increasing the load on the wireless channel, and so on. This snowball effect is illustrated in Figure 11, which depicts the amount of DENM messages transmitted by every node. It can be observed that a small change in EDCA parameters results in a dramatic increase of the number of transmitted DENM messages per node. It can hence be concluded that, when using forwarding optimizations that rely on CAM beacons to estimate the neighborhood density, it is beneficial to apply the same QoS class for both CAM and DENM messages. In that case, the optimal choice is to apply the VO class. Therefore, the configuration of both CAM and DENM messages as $\mathrm{VO}$ traffic is defined as the next key characteristic of SDDV (level 5).

\subsection{Adaptive beaconing}

It can be derived from Figure 11 that CAM beacons introduce a significant higher load on the wireless channel than DENM messages: during the course of the experiment, every node (except for DENM source nodes) transmits 350 created CAM messages but only 1 to 50 forwarded DENM messages. Therefore, it would be useful to reduce the amount of transmitted CAM messages. The most obvious technique to achieve this goal is a dynamic adjustment of the CAM beacon interval.

Some authors have already explored this technique before. The work of ElBatt et al. [57] simulated CAM beaconing scenarios with high vehicle density. Lowering the beaconing frequency from 10 to $5 \mathrm{~Hz}$ resulted in a significant increase of the CAM PSR. In [11], similar scenarios were researched, and equal conclusions could be drawn: $10 \mathrm{~Hz}$ resulted in problematic network performance, while no issues could be identified at $2 \mathrm{~Hz}$. Huang et al. [46] proposed to dynamically adjust the beaconing interval based on an estimation of the accuracy of the local dynamic map of the node's neighbors. Such a local dynamic map is constructed using information retrieved from the CAM messages and indicates the exact position of all neighbors in the node's vicinity. An estimation of the PSR is applied to estimate the accuracy of this local dynamic map. Some small scale outdoor tests were presented; however, they do not clearly indicate the actual impact under high vehicle densities. In [58], a similar technique was proposed: the beaconing interval was dynamically adjusted based on the channel quality. This metric combines channel collisions, SNR values on the 


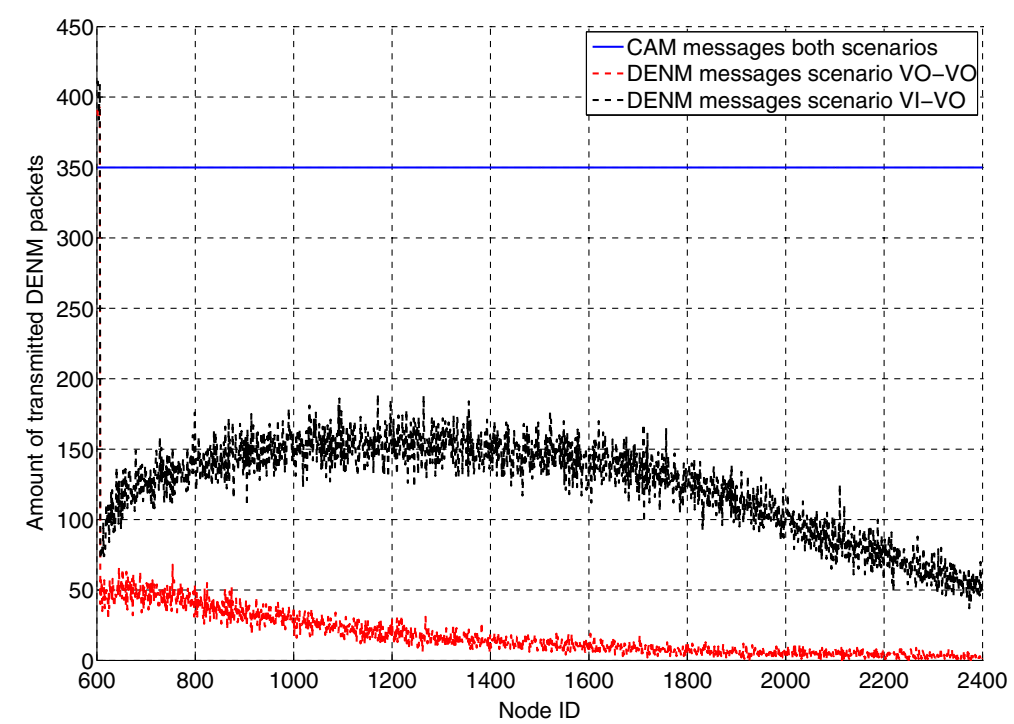

Figure 11 Distortion in the irresponsible forwarding scheme when assigning a lower QoS class to CAM traffic. When both traffic types are sent as VO traffic, every node only relays 50 or less DENM messages. When changing the CAM QoS class to VI (which is not that different from VO), the amount of transmitted DENM messages per node increases significantly.

channel, and neighborhood density. However, the proposed solution is designed for a relatively slow but reliable dissemination of non-safety related data within an often disconnected VANET. This is quite different from the scalability problem scenario studied in this paper.

Based on the positive results indicated in [11,57], it was concluded that adaptive beaconing should definitely be further explored. However, since a reduced level of complexity is pursued in every SDDV technique, a different approach was taken than proposed in $[46,58]$. Instead, we focused on the minimum CAM service level. Considering a maximum speed $v_{\max }$ of $130 \mathrm{~km} / \mathrm{h}$, in case of a fixed beaconing interval $I_{\text {static }}$ of $100 \mathrm{~ms}$, the maximum distance between two consecutive CAM transmissions is $3.6111 \mathrm{~m}$. We propose that based on the vehicle's speed, the node continuously adapt the beaconing interval $I_{\text {adaptive }}$ to guarantee this minimum CAM service level. In other words, the node will continuously re-calculate the appropriate beaconing interval in such a way that a CAM message is sent every $3.6111 \mathrm{~m}$. The formula to calculate the interval is as follows:

$$
I_{\text {adaptive }}=\frac{v_{\max }}{v} \times I_{\text {static }}
$$

Note that in this formula, $v$ is the current speed of the vehicle. We also remark that the DENM beaconing interval is not adjusted according to the vehicle speed. The motivation behind this design choice is the fact that CAM messages are intended for the exchange of vehicle position, speed, heading, etc. between vehicles in each others vicinity. Hence, the sender and intended receivers are part of the same traffic flow, which results in a relatively small speed difference between them. Therefore, the CAM service level as calculated by the sender node will correspond with the achieved service level at the intended receivers. However, in the case of DENM messages, it can often happen that messages from slow or stationary vehicles are intended for approaching high-speed receivers. In this case, adaptive DENM beaconing would result in a low beaconing frequency at the sender, hereby failing to reach the targeted data service level at the intended receivers.

The results of our adaptive CAM beaconing approach are depicted in Figure 12. It can be observed that in case of fluent traffic (160, 80, and $40 \mathrm{~m}$ inter-vehicle distance), the effects are rather limited. This is expected, since the average vehicle speed is not that much below $130 \mathrm{~km} / \mathrm{h}$ (see Table 2), and hence the dynamic beaconing intervals are approximately the same as in the case of static beaconing. However, in the case of a traffic jam (inter-vehicle space $10 \mathrm{~m}$ ), the speed of the vehicles is reduced to $25 \mathrm{~km} / \mathrm{h}$. This leads to significant benefits in terms of PSR and delay regarding both CAM and DENM traffic. Based on these positive results, adaptive beaconing of CAM messages is adopted as the sixth level of SDDV.

\subsection{Improved channel tracking using decision feedback and smoothing}

The IEEE $802.11 \mathrm{p}$ standard uses an orthogonal frequency division multiplexing (OFDM)-based communication scheme that is closely related to the IEEE 802.11a/g standards for wireless LAN. An essential part in such a receiver is channel estimations, which allows equalizing the OFDM signal. This is crucial for achieving a high 


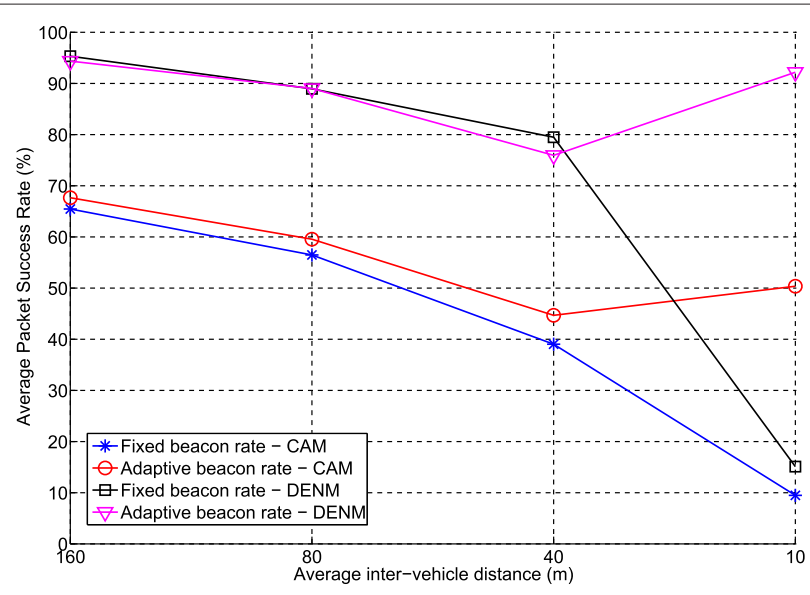

(a)

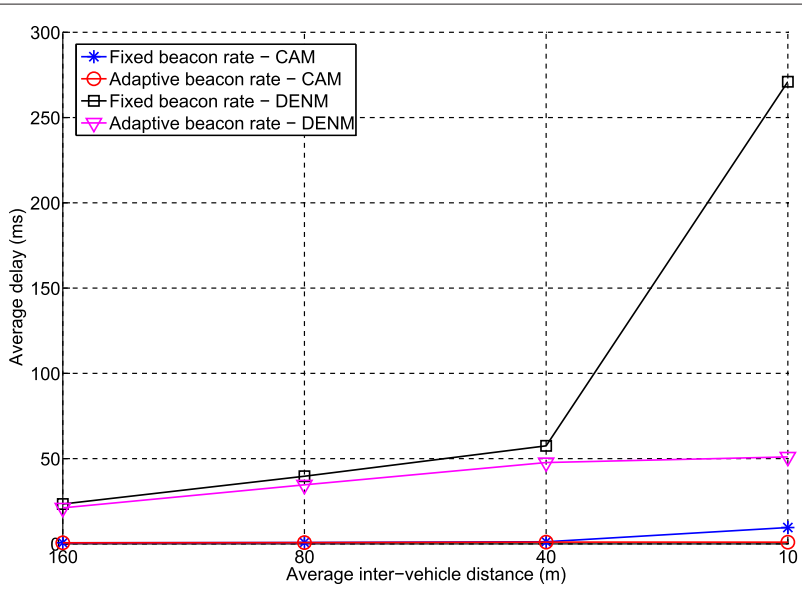

(b)

Figure 12 Effect of adaptive beaconing of CAM messages based on the velocity of the sender. In flowing traffic conditions, the added value of this technique is limited; in case of traffic jams (10 m inter-vehicle distance), the positive effect is significant. (a) Influence of adaptive beaconing on PSR. (b) Influence of adaptive beaconing on delay.

packet success ratio. In IEEE $802.11 \mathrm{a} / \mathrm{g} / \mathrm{p}$, most information to estimate the channel is coming from the initial part of a packet, more specifically the preamble. At urban and highway speeds, the channel varies much faster than in stationary applications. Also, insufficient explicit information is available in the signal to update the channel estimates. As a consequence, the amount of packet errors rises quickly in function of the packet length. Several techniques have been proposed to tackle this problem in a way that is compatible with the IEEE 802.11 p standard. Examples are decision feedback-based tracking [59] and smoothing, using a spectral temporal averaging estimation [60]. Recent progress in the state of the art shows that a combination of both decision feedback and smoothing provides an improved tracking performance compared to applying each technique separately. A further enhancement is the use of multiple antenna reception techniques. Our work focuses on the achievable signal processing gains of these proposed techniques [61]. The following approaches are adopted:

- Decision feedback and smoothing information: using the maximum amount of data carriers to derive the channel estimate results in the best performance. However, this also leads to a higher computational complexity overhead. A possible technique to reduce this computational complexity is to lower the amount of used data carriers. This results in a degradation of the accuracy of the channel estimation though. To achieve a significant decrease in computation complexity, the amount of data carriers has to be lowered in such a way that the corresponding performance decrease is unacceptable. Therefore, it is chosen to use all data carriers when estimating the channel based on decision feedback and smoothing information.

- A single antenna receiver: although the usage of multiple antenna receivers could introduce additional performance gains, it was chosen to adopt the single antenna approach. This design choice was driven by the corresponding cost increase. More antennas require more wiring in the car frame, which is a sensitive cost issue in the automotive sector.

These techniques were studied on the Matlab model of the IEEE 802.11p standard that was introduced in Section 2 and then applied in our NS-2 simulations. As a result, we could observe an improved packet success rate in the NS-2 simulations, as illustrated in Figure 13. The block effect that was present in our previous CAM plots has completely disappeared. As mentioned in Section 2, the simulated highway nodes are numbered sequentially from start to finish, and each successive ID corresponds with a node on the next lane. Therefore, there is a change of driving directions after every three consecutive node IDs. This explains the block effect: for a given sender, all receivers in the opposite direction will lose more packets due to node mobility, resulting in lower CAM PSR values. When applying decision feedback and smoothing, this difference disappears, and hence, the blocking effect is no longer present. As a result, the networking performance becomes more uniform, which is a desired characteristic for road safety applications. Therefore, the implementation of improved channel tracking using decision feedback and smoothing is defined as the next key characteristic of SDDV (level 7). 


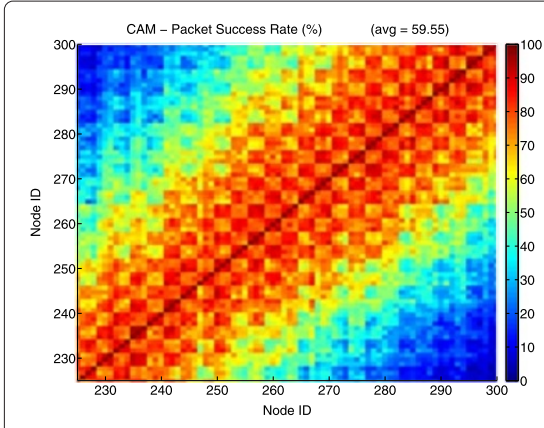

(a)

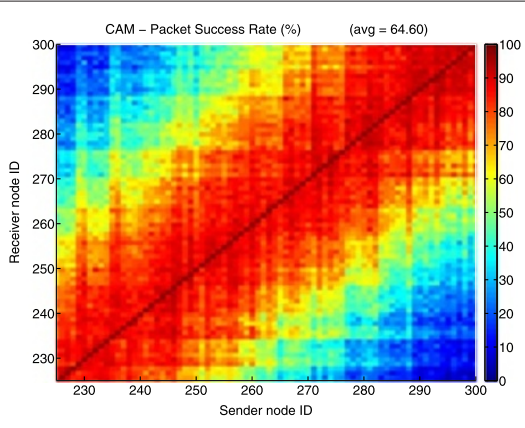

(b)

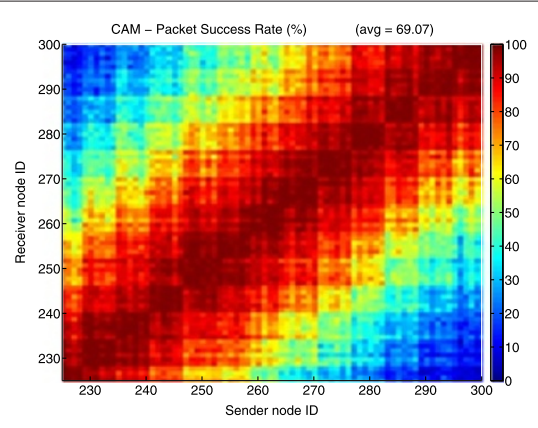

(c)

Figure 13 Effect of channel tracking using decision feedback and smoothing and of preamble and data switching. The former solves the block effect that characterized CAM PSR plots until now. The latter results in a much thicker dark red diagonal. This means that CAM dissemination from nodes to their most immediate neighbors becomes more reliable. (a) Standard IEEE 802.11 p PHY layer. (b) + decision feedback and smoothing. (c) + preamble and data switching.

\subsection{Preamble and data switching}

Other possible PHY optimizations are preamble and data switching. They are explained in detail in [22]. In short, preamble switching occurs when a new frame arrives at the time that the receiver node is still receiving the preamble and physical layer convergence procedure (PCLP) header of an earlier frame. The new frame can be picked and locked onto by the receiver node if it has sufficiently higher power to be heard above the earlier one. Data switching refers to the capture of a new incoming frame during the frame body reception process of another one. The result of applying these techniques on top of SDDV level 7 is depicted in part (c) of Figure 13. It can be observed that in the CAM PSR plot, a darker red area has appeared around the diagonal. This means that the CAM PSR for a node's most direct neighbors has reached very high values. This is a desirable VANET communication characteristic. Therefore, we define the adoption of preamble and data switching as the last key characteristic of SDDV (level 8).

\section{Final SDDV specification}

In the previous section, SDDV was gradually composed. A summary of all elements included in the final SDDV specification is given in Table 1 (introduced in Section 1). The corresponding performance characteristics are depicted in Figures 14 and 15. It can be observed that the scalability issues which were clearly visible in the evaluation of the baseline solution in Section 3 have completely disappeared in SDDV. For all envisaged traffic intensities, the CAM PSR plots indicate that these beacons can be reliably disseminated to all nodes within a range of at least $300 \mathrm{~m}$. The corresponding delay values are very small, with an average of $1 \mathrm{~ms}$ or lower in all traffic conditions. DENM values only show a light descent over a length of
$3 \mathrm{~km}$, which results in very predictable DENM networking performance. In the worst case (intense but fluent traffic, $40 \mathrm{~m}$ inter-vehicle distance), the minimum PSR at a distance of $3 \mathrm{~km}$ is still $60 \%$, in all other cases, it is $80 \%$ or more. This means that at this distance, vehicles will at least receive six DENM messages per second from a given source. Without any doubt, this is sufficient to allow timely warning of the driver. In terms of delay, the maximum delay is $100 \mathrm{~ms}$ under all circumstances. This complies with the maximum acceptable DENM delay defined by ETSI [30]. Based on these simulation results, it can be concluded that SDDV achieves its goal of solving the VANET scalability problem by defining a specific combination of optimization techniques which are spread across the different layers of the OSI protocol stack.

\section{Experimental validation on the iMinds w-iLab.t wireless testbed}

Now that the performance of SDDV has been evaluated in the NS-2 simulator, the next step in our research is to validate SDDV on actual hardware. For this task, we made use of the iMinds w-iLab.t wireless testbed, which was introduced in Section 2. As discussed in our previous work [23], the third floor of this testbed is a useful environment to validate VANET solutions. It consists of 110 nodes distributed in a highway-alike topology (Figure 16, the 55 physical nodes are each equipped with 2 wireless interfaces; an independent VANET stack runs on each of them). Although the nodes are equipped with IEEE 802.11a hardware, the necessary software adjustments were deployed to approximate IEEE 802.11p characteristics [51]. When applying the maximum transmission power $(23 \mathrm{dBm})$ and the $12 \mathrm{Mbps}$ transmission rate, approximately 50 nodes are within a single node's 


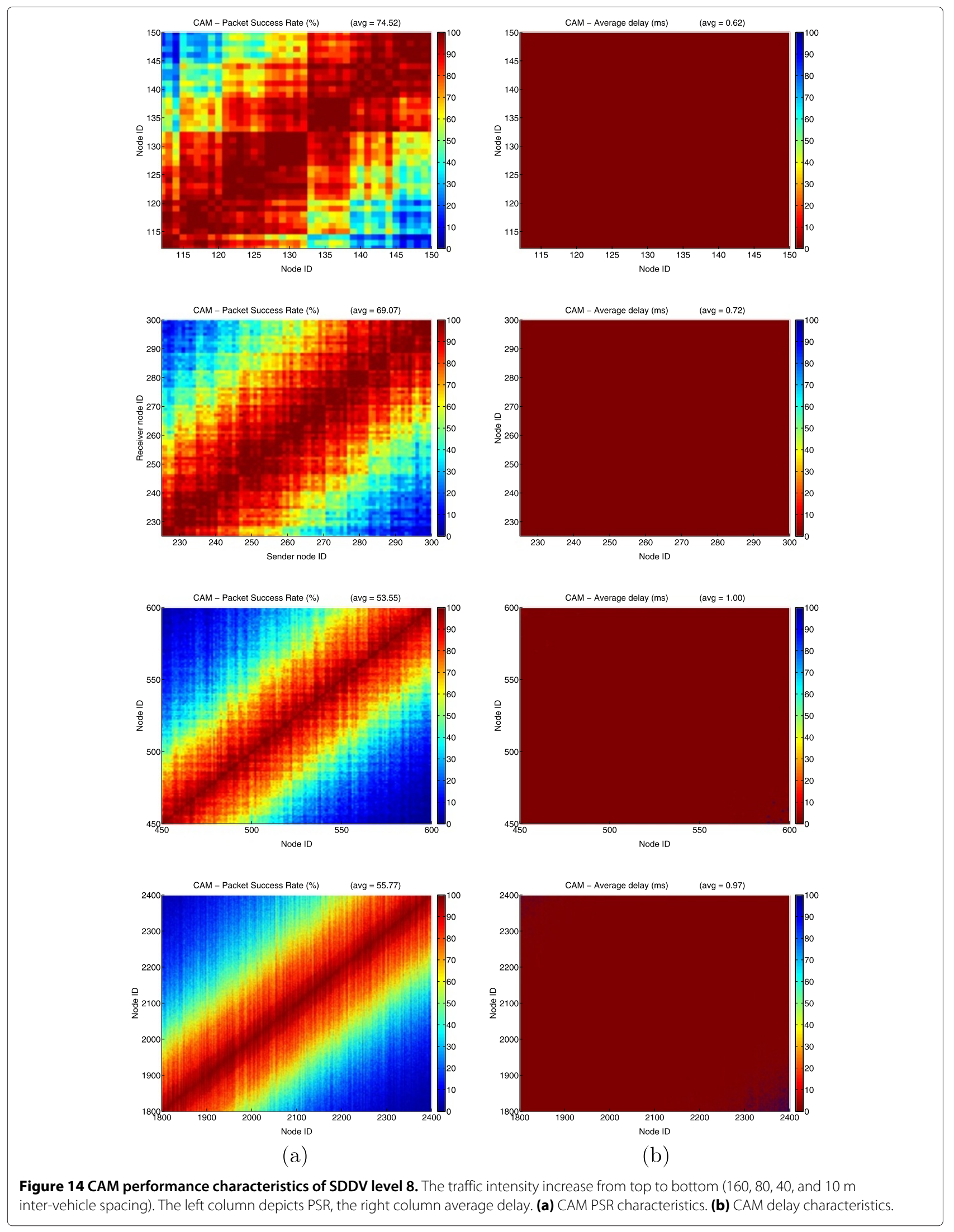



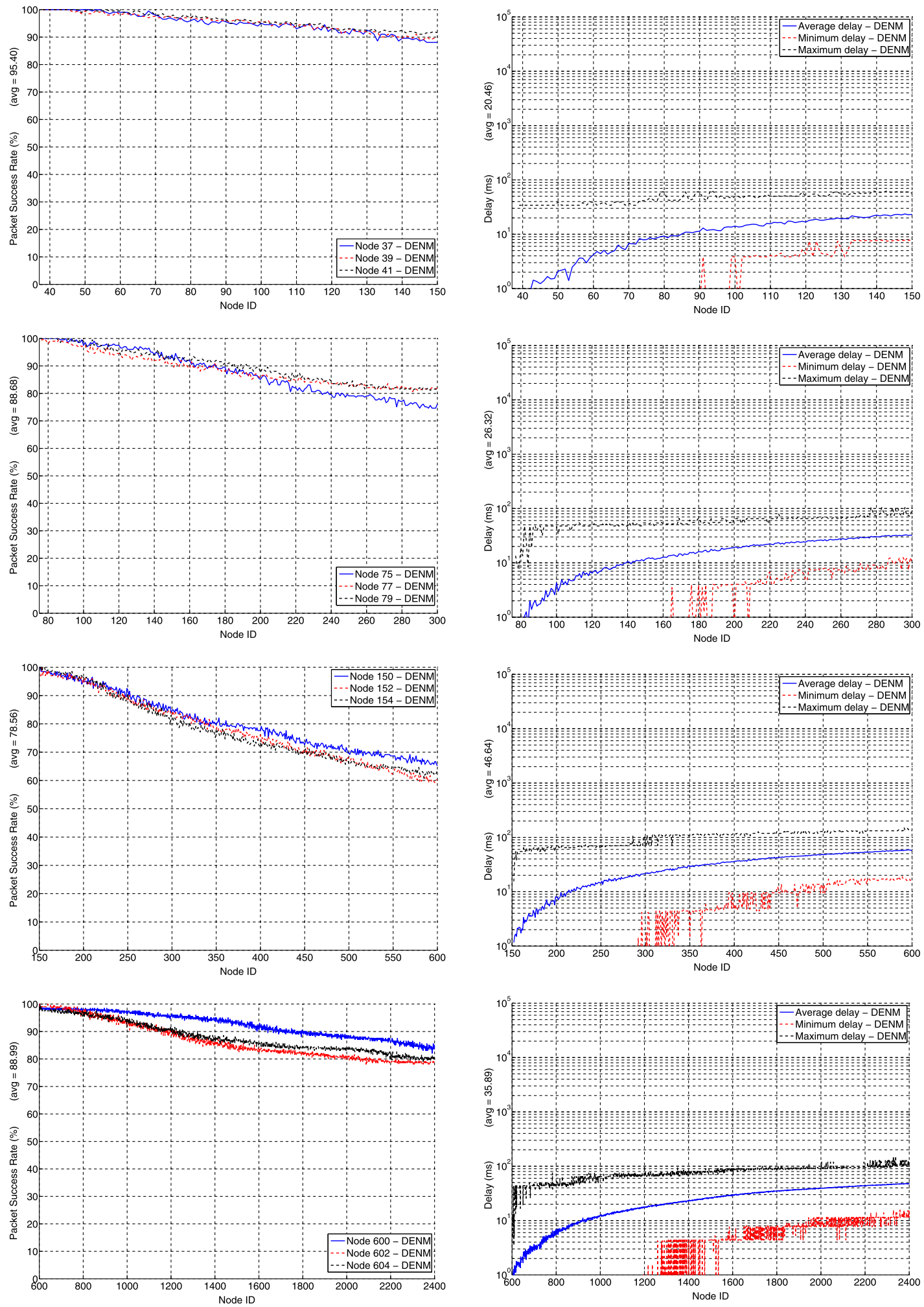

(a)

(b)

Figure 15 DENM performance characteristics of SDDV level 8. The traffic intensity increase from top to bottom (160, 80, 40, and 10 m inter-vehicle spacing). The left column depicts PSR, the right column average delay. (a) DENM PSR characteristics. (b) DENM delay characteristics. 


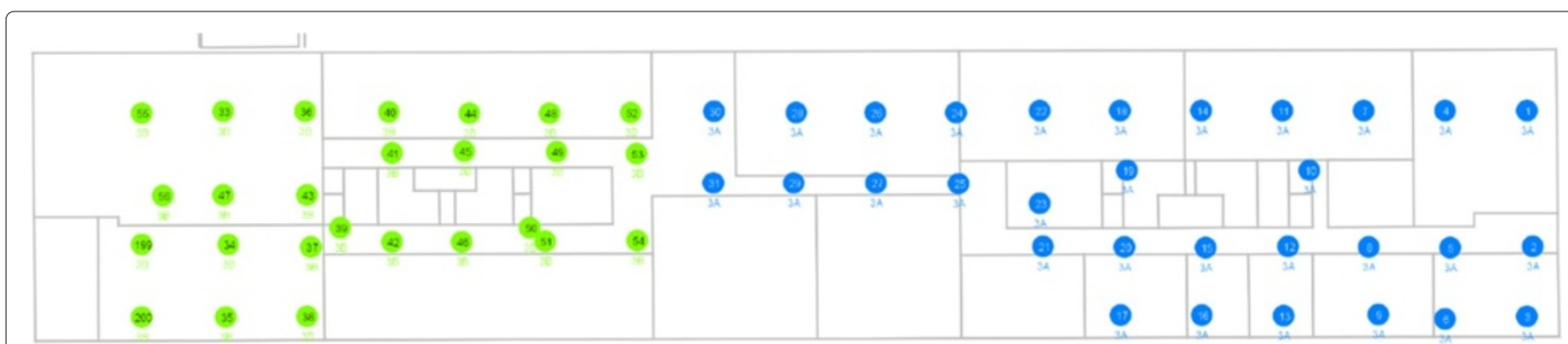

Figure 16 Topology of the iMinds w-iLab.t wireless lab (third floor). There are 55 physical nodes, each equipped with two wireless interfaces. An independent VANET stack runs on each of them. Hence, 110 VANET nodes participate in the experiment.

transmission domain. This node density corresponds with an inter-vehicle distance between 160 and $80 \mathrm{~m}$.

Two experiments were performed: the baseline solution presented in Section 3 and SDDV level 8. In both experiments, the scenario was kept similar to the simulation scenario: six nodes located at one end of the building are DENM sources, all nodes forward these messages. In parallel, all nodes transmit CAM messages of their own. The same timing is adopted as in the simulations: $10 \mathrm{~s}$ warm-up, $20 \mathrm{~s}$ measurements, $5 \mathrm{~s}$ cool-down. To calculate accurate delay values, the clocks of all nodes were synchronized using the PTPd protocol [62]. It was verified that during the experiments, the accuracy of the clock synchronization was always below $0.01 \mathrm{~ms}$.

To the best of our knowledge, it is the first time that such an experiment has been conducted in the context of the VANET scalability problem. The only related study is the work of Ramachandran et al. [8] which was performed on the ORBIT testbed. However, (1) these experiments included only CAM beaconing, not DENM forwarding; (2) there was no approximation of the IEEE 802.11p standard; and (3) the packet size was 128 bytes instead of 300 bytes. An overview of other VANET research laboratories is given in [63]. None of them can support experiments on the same scale as iMinds w-iLab.t or ORBIT.

The results of our experiment are depicted in Figure 17. As can be expected, they are not exactly the same as in any of the simulation results. This is because the topology is not as symmetric as in the simulation; the indoor location leads to different propagation characteristics; the presence of concrete walls and elevator shafts can block communication between nearby nodes; and the emulated inter-vehicle distance is not equal to any of the four simulated ones. However, the effect of SDDV compared to the baseline scenario clearly follows the same trends as the simulations of the inter-vehicle distances that corresponds most with the testbed environment (160 and $80 \mathrm{~m})$. The improvement in CAM PSR is significant. Note that the PSR plot corresponds with calibration measurements of the testbed; hence, when nodes cannot communicate, this is caused by signal attenuation and not as a result of the scalability problem. The average CAM delay is also drastically reduced. In terms of DENM PSR, there is only a slight increase. However, there was almost no room for improvement since the vehicle density was not high enough to introduce major DENM PSR issues in the baseline solution. This behavior was also observed in our simulations (see Figure 4). The average DENM delay was reduced with a factor 2.5 , which again is a significant improvement.

A peculiar effect worth mentioning is the fact that SDDV introduces two dips in the DENM PSR curves. Such a behavior could not be observed in any of our simulations. The cause of this effect is the location of the corresponding nodes: they are positioned inside concrete maintenance shafts that interconnect the different floors of the building. Hence, the signals sent to these nodes are attenuated more fiercely. In the baseline solution, no problems occur due to the high level of rebroadcasting redundancy of the simple flooding technique. However, the reduced redundancy level of SDDV fails to entirely overcome this problem. This means that in real-life situations where some vehicles are enclosed by obstacles (e.g., trucks), it might be required to tweak the SDDV parameters to achieve reliable dissemination to these nodes without sacrificing general VANET performance. This can be researched in future work.

\section{Conclusions}

This paper focused on the VANET issues regarding scalable data dissemination. Simulations have illustrated the severity of this problem when adopting common techniques and parameter values. Step by step, optimizations were combined to compose a holistic solution: SDDV. During the selection of each possible technique, consideration was given to its degree of complexity. The final result is a composition of eight techniques: opportunistic forwarding, irresponsible forwarding, fixed transmit power of $33 \mathrm{dBm}$, fixed transimission rate of $12 \mathrm{Mbps}$, adoption of the VO QoS class for both CAM and DENM messages, adaptive beaconing based on the vehicle speed, improved 

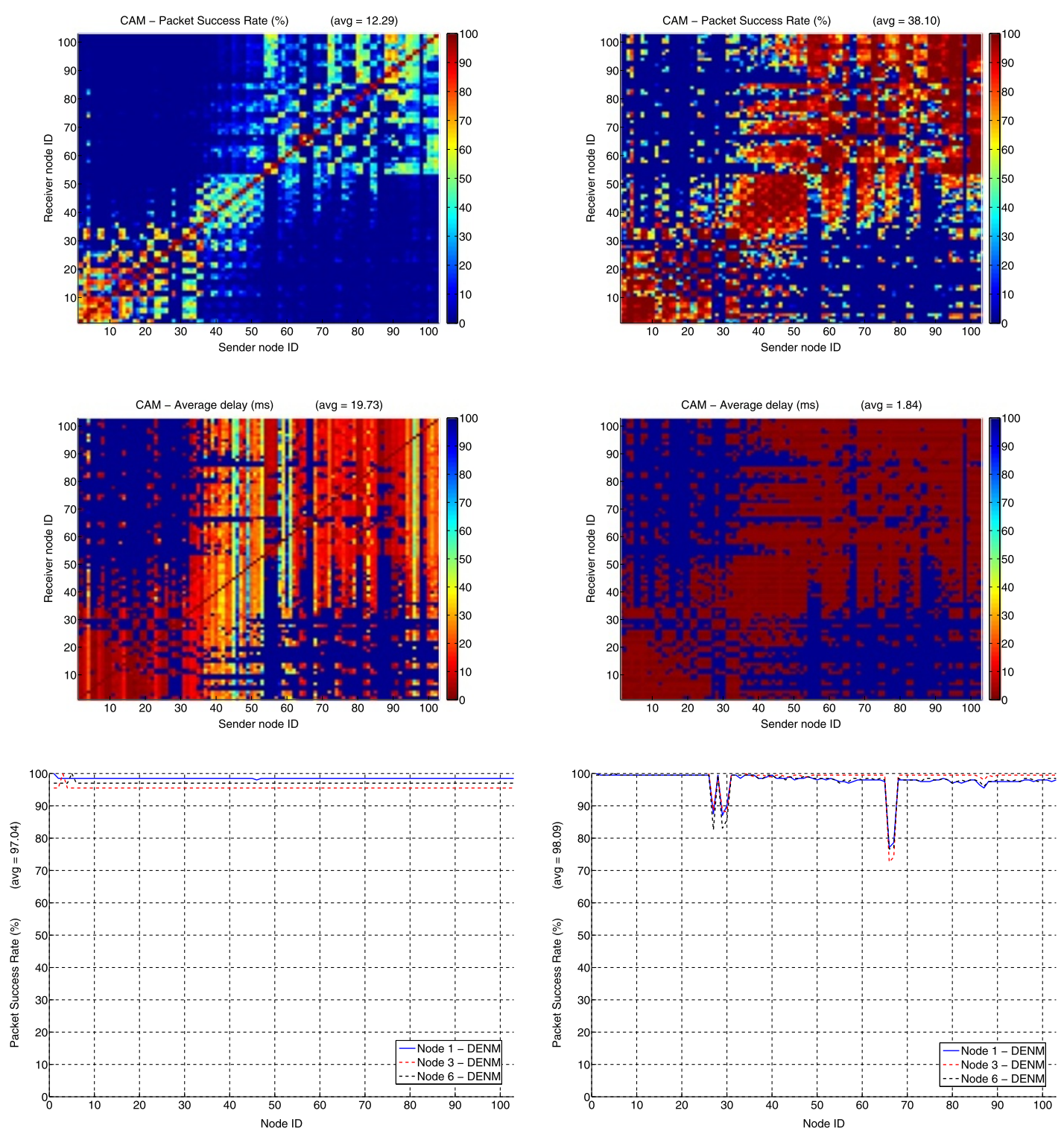

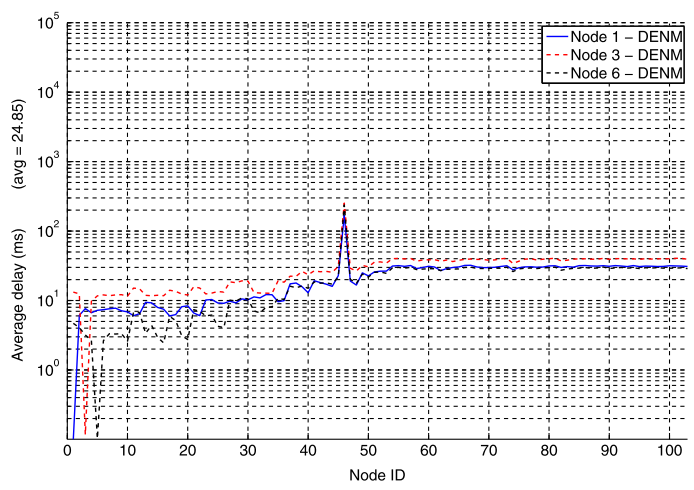

(a)

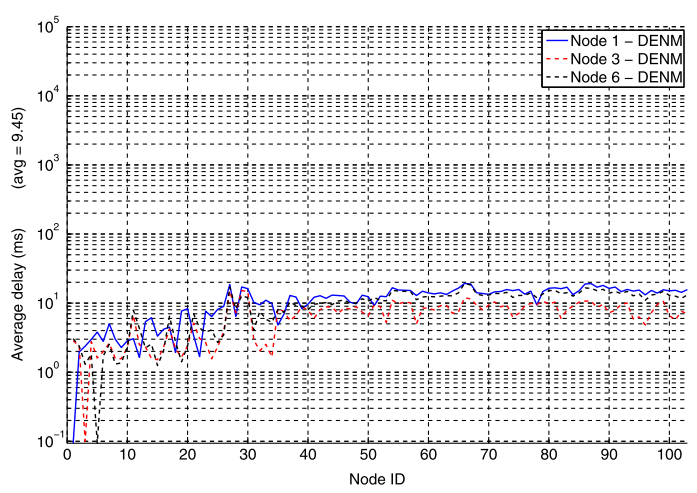

(b)

Figure 17 Performance comparison between the baseline VANET solution and SDDV level 8, as retrieved from the experiments performed on the iMinds w-iLab.t wireless testbed. Observed trends are similar to the simulated scenarios which correspond most with the testbed environment (160 and $80 \mathrm{~m}$ ). (a) Baseline solution. (b) SDDV level 8. 
channel tracking using decision feedback and smoothing, and preamble and data switching.

Through simulation, it was evaluated that SDDV achieves its goal of providing scalable VANET data dissemination under all types of traffic intensities in the highway environment. A final validation of SDDV was performed on the iMinds w-iLab.t wireless testbed. This experiment confirmed the simulated results. However, some important topics for future work can be identified. The w-iLab.t experiment indicated that some optimization of SDDV might be required in case of vehicle obstruction. Another topic is the analytical analysis of SDDV. The holistic approach pursued in this paper led to a large research space to explore. The empirical approach adopted in this paper was considered to be the only practical way to work towards the definition of SDDV. Performing an analytical analysis of every intermediate step and every possible combination of techniques seemed unfeasible. However, now that SDDV has been defined, it would be very useful to analyze it analytically. Similar to the approach taken to validate SDDV on the iMinds w-iLab.t wireless testbed, a theoretical comparison of the baseline solution and SDDV would be vary valuable. Another possibility for the further improvement of SDDV is to extend the currently targeted highway environment with the urban setting. In our research, we focused on the highway scenario because this is the case where the scalability problem is the most apparent. It is challenging for the IEEE $802.11 \mathrm{p}$ signal to penetrate buildings. As a result, the urban environment is actually characterized by a lower amount of competing nodes on the wireless channel, although there is actually a higher vehicle density in the city then on the highway. Hence in this paper, it was shown that SDDV can handle the most challenging scenario in terms of scalability, and it is expected to be evenly successful in less challenging urban environments. In future work, a validation of this assumption would be valuable. If needed, SDDV could be further extended in this case with techniques that intelligently cope with forwarding decisions at intersections. Another possible direction for future work is the validation of SDDV in large-scale field operational tests (FOT). Since SDDV was deliberately designed using techniques characterized by a rather low level of complexity, it seems feasible to adopt it in any FOT within reasonable constraints regarding manpower and budget. Such a valuable validation of SDDV in a real-life circumstances should therefore be pursued in future work.

\section{Competing interests}

The authors declare that they have no competing interests.

\section{Author details}

${ }^{1}$ iMinds, Department of Information Technology (INTEC), Ghent University, Gent, Belgium. ${ }^{2}$ imec, Leuven, Belgium.
Received: 29 November 2013 Accepted: 7 October 2014

Published: 4 November 2014

\section{References}

1. Wireless LAN Medium Access Control (MAC) and Physical Layer (PHY) specifications - Amendment 6: Wireless Access in Vehicular Environments. IEEE Standard $802.11 \mathrm{p}-2010$ (2010)

2. Intelligent Transport Systems; Vehicular Communications; Basic Set of Applications; Part 2: Specification of Cooperative Awareness Basic Service, ETSI standard TS 102 637-2 (2010)

3. Intelligent Transport Systems; Vehicular Communications; Basic Set of Applications; Part 2: Specification of Decentralized Environment Notification Basic Service, ETSI standard TS 102 637-3 (2010)

4. S Eichler, in Proc. IEEE 65th Vehicular Technology Conf. Fall. Performance evaluation of the IEEE 802.11p WAVE communication standard (Baltimore, MD, USA, Sept. 2007), pp. 2199-2203

5. K Bilstrup, E Uhlemann, EG Ström, U Bilstrup, On the ability of the $802.11 \mathrm{p}$ MAC method and STDMA to support real-time vehicle-to-vehicle communication. EURASIP J. Wirel. Comm. 2009(902414) (2009)

6. N Wisitpongphan, OK Tonguz, JS Parikh, P Mudalige, F Bai, V Sadekar, Broadcast storm mitigation techniques in vehicular ad hoc networks. IEEE Wireless Commun. 14(6), 84-94 (2007)

7. H Yomo, O Shagdar, T Ohyama, M Miyamoto, Y Kondo, J Hasegawa, T Sakai, R Miura, S Obana, Development of a CDMA intervehicle communications system for driving safety support. IEEE Wireless Commun. 16(6), 24-31 (2009)

8. KRamachandran, M Gruteser, R Onishi, T Hikita, Experimental analysis of broadcast reliability in dense vehicular networks. IEEE Veh. Technol. Mag. 2(4), 26-32 (2007)

9. X Ma, X Chen, IEEE 802.11 broadcast scheme in ad hoc wireless LANs. IEEE Trans. Veh. Technol. 57(6), 3757-3768 (2008)

10. MI Hassan, HL Vu, T Sakurai, Performance analysis of the IEEE 802.11 MAC protocol for DSRC safety applications. IEEE Trans. Veh. Technol. 60(8), 3882-3896 (2011)

11. XMa, J Zhang, TWu, Reliability analysis on one-hop safety-critical broadcast services in VANETs. IEEE Trans. Veh. Technol. 60(8), 3933-3946 (2011)

12. Y Kim, K Kim, S Pack, W Lee, Analysis of session handover probability in NEMO-based vehicular networks. Springer Wireless Pers. Commun. 61(4), 697-710 (2011)

13. J-H Lee, T Ernst, Lightweight network mobility within PMIPv6 for transportation systems. IEEE Syst. J. 5(3), 352-361 (2011)

14. J-H Lee, T Ernst, N Chilamkurti, Performance analysis of PMIPv6 based network mobility for intelligent transportation systems. IEEE Trans. Veh. Technol. 61(1), 74-85 (2012)

15. I Ivan, P Besnier, M Crussiere, M Drissi, L Le Danvic, M Huard, E Lardjane, in Proc. 9th Int. Conf. Intelligent Transport Systems Telecommunications. Physical layer performance analysis of V2V communications in high velocity context (Lille, France, Oct. 2009), pp. 409-414

16. W Zhang, A Festag, R Baldessari, L Le, in Proc. 8th Int. Conf. ITS Telecommunications. Congestion control for safety messages in VANETs: concepts and framework (Thailand Phuket, Oct. 2008), pp. 199-203

17. RK Schmidt, A Brakemeier, T Leinmüller, B Boddeker, G Schafer, in Proc. 10th Int. Conf. Intelligent Transport Systems Telecommunications. Architecture for decentralized mitigation of local congestion in VANETs (Kyoto, Japan, Nov. 2010)

18. F Dressler, F Kargl, J Ott, OK Tonguz, L Wischhof, Research challenges in intervehicular communication: lessons of the 2010 Dagstuhl Seminar. IEEE Commun. Mag. 49(5), 158-164 (2011)

19. R Baldessari, D Scanferla, L Le, W Zhang, A Festag, in Proc. 7th Int. Workshop Intelligent Transportation. Joining forces for VANETs: a combined transmit power and rate control algorithm (Hamburg, Germany, Mar. 2010)

20. S Bouckaert, W Vandenberghe, B Jooris, I Moerman, P Demeester, in Proc. 6th Int. Conf. Testbeds and Research Infrastructures for the Development of Networks and Communities. The w-iLab.t testbed (Berlin, Germany, May 2010), pp. 145-154

21. The Network Simulator - ns-2. Available: http://www.isi.edu/nsnam/ns

22. Q Chen, F Schmidt-Eisenlohr, D Jiang, M Torrent-Moreno, L Delgrossi, H Hartenstein, in Proc. 10th ACM Int. Symp. Modeling, Analysis and Simulation of Wireless and Mobile Systems. Overhaul of IEEE 802.11 modeling and simulation in NS-2 (Chania, Greece, Oct. 2007), pp. 159-168 
23. W Vandenberghe, I Moerman, P Demeester, H Cappelle, in Proc. SCVT. Suitability of the wireless testbed w-iLab.t for VANET research, vol. 2011 (Ghent, Belgium, Nov. 2011), pp. 1-6

24. E Kohler, The Click modular router. Ph.D. dissertation, Massachusetts Institute of Technology (2000)

25. W Vandenberghe, E Van de Velde, C Blondia, I Moerman, P Demeester, Vehicular ad hoc networking based on the incorporation of geographical information in the IPv6 header. EURASIP J. Wirel. Comm. revision under review

26. Intelligent Transport Systems - Communications access for land mobiles (CALM) - Non-IP networking, ISO standard DIS 29281 (2009)

27. Intelligent Transport Systems (ITS); Vehicular Communications; GeoNetworking; Part 4: Geographical Addressing and Forwarding for Point-to-Point and Point-to-Multipoint Communications; Sub-part 1: Media Independent Functionalities, ETSI TS 102 636-4-1 (2011)

28. D Jiang, Q Chen, L Delgrossi, in Proc. 5th Int. Workshop on Vehicular Inter-Networking. Optimal data rate selection for vehicle safety communications (San Fransisco, CA, USA, Sept. 2008), pp. 30-38

29. CAR 2 CAR Communication Consortium, CAR 2 CAR Communication Consortium Manifesto (2007). https://www.car-2-car.org/.

30. Intelligent Transport Systems; Vehicular Communications; Basic Set of Applications; Definitions. ETSI TR 102638 (2009)

31. MN Mariyasagayam, T Osafune, M Lenardi, in Proc. 7th Int. Conf. on ITS Telecommunications. Enhanced multi-hop vehicular broadcast (MHVB) for active safety applications (Sophia Antipolis, France, June 2007), pp. 1-6

32. B Blaszczyszyn, A Laouiti, P Muhlethaler, Y Toor, in Proc. 8th Int. Conf. ITS Telecommunications. Opportunistic broadcast in VANETs (OB-VAN) using active signaling for relays selection (Phuket, Thailand, Oct. 2008), pp. 384-389

33. V Naumov, R Baumann, T Gross, in Proc. 7th ACM Int. Symp. Mobile Ad Hoc Networking and Computing. An evaluation of inter-vehicle ad hoc networks based on realistic vehicular traces (Florence, Italy, May 2006), pp. 108-119

34. K Ibrahim, MC Weigle, M Abuelela, in Proc. IEEE 69th Vehicular Technology ConfAbuelela, M. p-IVG: Probabilistic inter-vehicle geocast for dense vehicular networks (Barcelona, Spain, Apr. 2009), pp. 1-5

35. J Nzouonta, N Rajgure, GG Wang, C Borcea, VANET routing on city roads using real-time vehicular traffic information. IEEE Trans. Veh. Technol. 58(7), 3609-3626 (2009)

36. M Nekovee, Epidemic algorithms for reliable and efficient information dissemination in vehicular ad hoc networks. IET Intell. Transp. Sy. 3(2), 104-110 (2009)

37. S Panichpapiboon, G Ferrari, in Proc. 8th Int. Conf. ITS Telecommunications. Irresponsible forwarding (Phuket, Thailand, Oct. 2008), pp. 311-316

38. S Busanelli, G Ferrari, S Panichpapiboon, in Proc. IEEE Global Communications Conf. Efficient broadcasting in IEEE 802.11 networks through irresponsible forwarding (Honolulu, HI, USA, Nov. 2009), pp. 1-6

39. OK Tonguz, N Wisitpongphan, F Bai, DV-CAST: a distributed vehicula broadcast protocol for vehicular ad hoc networks. IEEE Wireless Commun. 17(2), 47-56 (2010)

40. Y Mylonas, M Lestas, A Pitsillides, in Proc. 4th Int. Wireless Internet Conf. Speed adaptive probabilistic flooding in cooperative emergency warning (Maui, HI, USA, Nov. 2008), pp. 1-8

41. S Oh, J Kang, M Gruteser, in Proc. 3rd Ann. Int. Conf. Mobile and Ubiquitous Systems: Networks and Services. Location-based flooding techniques for vehicular emergency messaging (San Jose, CA, USA, July 2006)

42. M Torrent-Moreno, J Mittag, P Santi, H Hartenstein, Vehicle-to-vehicle communication: fair transmit power control for safety-critical information. IEEE Trans. Veh. Technol. 58(7), 3684-3703 (2009)

43. J Mittag, F Schmidt-Eisenlohr, M Killat, J Härri, H Hartenstein, in Proc. 5th ACM Int. Workshop Vehicular Inter-networking. Analysis and design of effective and low-overhead transmission power control for VANETs (San Fransisco, CA, USA, Sept. 2008), pp. 39-48

44. M Artimy, Local density estimation and dynamic transmission-range assignment in vehicular ad hoc networks. IEEE Trans. Intell. Trans. Syst. 8(2), 400-412 (2007)

45. G Caizonne, in Proc. 2005 IEEE Int. Conf. Communications. A power control algorithm with high channel availability for vehicular ad hoc networks (Seoul, Korea, May 2005), pp. 3171-3176
46. CL Huang, R Sengupta, H Krishnan, YP Fallah, Implementation and evaluation of scalable vehicle-to-vehicle safety communication control. IEEE Commun. Mag. 49(11), 134-141 (2011)

47. P Shankar, T Nadeem, J Rosca, L Iftode, in Proc. IEEE Int. Conf. Network Protocols. CARS context-aware rate selection for vehicular networks (Orlando, FL, USA, Oct. 2008), pp. 1-12

48. K Wang, F Yang, Q Zhang, DO Wu, Y Xu, Distributed cooperative rate adaption for energy efficiency in IEEE 802.11-Based multihop networks. IEEE Trans. Veh. Technol. 56(2), 888-898 (2007)

49. Q Xu, T Mak, J Ko, R Sengupta, Medium access control protocol design for vehicle-vehicle safety messages. IEEE Trans. Veh. Technol. 56(2), 499-518 (2007)

50. Wireless LAN Medium Access Control (MAC) and Physical Layer (PHY) specifications - Amendment 8: Medium Access Control (MAC) Quality of Service Enhancements, IEEE Standard 802.11 e-2005 (2005)

51. W Vandenberghe, I Moerman, P Demeester, in Proc. 11th Int. Conf. ITS Telecommunications. Approximation of the IEEE $802.11 \mathrm{p}$ standard using commercial off-the-shelf IEEE 802.11a hardware (Saint-Petersburg, Russia, Aug. 2011), pp. 21-26

52. M Torrent-Moreno, D Jiang, H Hartenstein, in Proc. First ACM Workshop Vehicular Ad Hoc Networks. Broadcast reception rates and effects of priority access in 802.11-based vehicular ad-hoc networks (Philadelphia, PA, USA, Oct. 2004), pp. 10-18

53. K Bilstrup, E Uhlemann, EG Ström, U Bilstrup, in Proc. 16th World Congr. on ITS. On the ability of IEEE 802.11p and STDMA to provide predictable channel access (Stockholm, Sweden, Sept. 2009), pp. 1-10

54. AR Rebai, S Hanafi, H Alnuweiri, in Proc. 9th Int. Conf. Intelligent Transport Systems Telecommunications. A new inter-node priority access enhancement scheme for IEEE_802.11 WLANs (Lille, France, Oct. 2009), pp. 520-525

55. C Adler, S Eichler, T Kosch, C Schroth, M Strassberger, in Proc. 3rd Int. Symposium Wireless Communication Systems. Self-organized and context-adaptive information diffusion in vehicular ad hoc networks (Valencia, Spain, Sept. 2006), pp. 307-311

56. DJ Deng, $\mathrm{HC}$ Chen, HC Chao, YM Huang, A collision alleviation scheme for IEEE 802.11p VANETs. Wireless Pers. Commun. 56(3), 371-383 (2011)

57. T ElBatt, SK Goel, G Holland, H Krishnan, J Parikh, in Proc. 3rd Int. Workshop Vehicular Ad Hoc Networks. Cooperative collision warning using dedicated short range wireless communications (Los Angeles, CA, USA, Sept. 2006), pp. 1-9

58. C Sommer, OK Tonguz, F Dressler, Traffic information systems: efficient message dissemination via adaptive beaconing. IEEE Commun. Mag. 49(5), 173-179 (2011)

59. J Ran, R Grunheid, H Rohling, E Bolinth, R Kern, in Proc. 57th IEEE Vehicular Technology Conf. Decision-directed channel estimation method for OFDM systems with high velocities (Jeju, Korea, Apr. 2003), pp. 2358-2361

60. JA Fernandez, DD Stancil, Fan Bai, in Proc. 48th Ann. Allerton Conf. Communication, Control and Computing. Dynamic channel equalization for IEEE 802.11p waveforms in the vehicle to vehicle channel (Monticello, IL, USA, Sept. 2010), pp. 542-551

61. A Bourdoux, H Cappelle, A Dejonghe, in Proc 2011 IEEE Global Communications Conf. Channel tracking for fast time-varying channels in, IEEE802.11p systems (Houston, TX, USA, Dec. 2011), pp. 1-6

62. T-K Want, F-R Chang, S-Y Lin, Multidevice time measurement system via a PTPD network. IEEE Trans. Instrum. Meas. 60(7), 2304-2307 (2011)

63. JA Dias, JN Isento, BMC Silva, VNG Soares, JJPC Rodrigues, Performance assessment of IP over vehicular delay-tolerant networks through the VDTN@Lab testbed. EURASIP J. Wirel. Comm. 2012, 13 (2012)

doi:10.1186/1687-1499-2014-182

Cite this article as: Vandenberghe et al:: SDDV: scalable data dissemination in vehicular ad hoc networks. EURASIP Journal on Wireless Communications and Networking 2014 2014:182. 